\title{
Nice reflection arrangements
}

\section{Torsten Hoge}

Fakultät für Mathematik und Physik

Leibniz Universität Hannover

30167 Hannover, Germany

hoge@math.uni-hannover.de

\author{
Gerhard Röhrle *
}

Fakultät für Mathematik

Ruhr-Universität Bochum

44780 Bochum, Germany

gerhard.roehrle@rub.de

Submitted: Jun 15, 2015; Accepted: Mar 29, 2016; Published: Apr 15, 2016

Mathematics Subject Classifications: 20F55, 52B30, 52C35, 14N20

\begin{abstract}
The aim of this note is a classification of all nice and all inductively factored reflection arrangements. It turns out that apart from the supersolvable instances only the monomial groups $G(r, r, 3)$ for $r \geqslant 3$ give rise to nice reflection arrangements. As a consequence of this and of the classification of all inductively free reflection arrangements from Hoge and Röhrle (2015) we deduce that the class of all inductively factored reflection arrangements coincides with the class of all supersolvable reflection arrangements. Moreover, we extend these classifications to hereditarily factored and hereditarily inductively factored reflection arrangements.
\end{abstract}

Keywords: Complex reflection groups, reflection arrangements, factored arrangements, inductively factored arrangements

\section{Introduction}

Let $\mathbb{K}$ be a field and let $V=\mathbb{K}^{\ell}$. Let $\mathcal{A}=(\mathcal{A}, V)$ be a central $\ell$-arrangement of hyperplanes in $V$ and let $L(\mathcal{A})$ be its intersection lattice. Let $\pi=\left(\pi_{1}, \ldots, \pi_{s}\right)$ be a partition of $\mathcal{A}$. Then $\pi$ is called nice for $\mathcal{A}$ or a factorization of $\mathcal{A}$ if roughly speaking it partitions $\mathcal{A}$ into mutually linearly independent sets and these are compatible with the intersection lattice $L(\mathcal{A})$, see Definition 2.12 below.

In 1992, Terao [Ter92] introduced the notion of a nice or factored arrangement to provide a necessary and sufficient condition for the Orlik-Solomon Algebra $A(\mathcal{A})$ of $\mathcal{A}$ to admit a tensor factorization as a graded $\mathbb{K}$-vector space. More precisely, let $\left[\pi_{i}\right]$ be the

*We acknowledge support from the DFG-priority program SPP1489 "Algorithmic and Experimental Methods in Algebra, Geometry, and Number Theory". 
$\mathbb{K}$-subspace of $A(\mathcal{A})$ spanned by 1 and the generators of $A(\mathcal{A})$ corresponding to $\pi_{i}$. In [Ter92, Thm. 2.8] (cf. [OT92, Thm. 3.87]), Terao proved that the map

$$
\kappa:\left[\pi_{1}\right] \otimes \cdots \otimes\left[\pi_{s}\right] \rightarrow A(\mathcal{A})
$$

given by multiplication is an isomorphism of graded $\mathbb{K}$-vector spaces if and only if $\pi$ is nice for $\mathcal{A}$, see Theorem 2.14 below. As a consequence, if $\pi=\left(\pi_{1}, \ldots, \pi_{s}\right)$ is nice for $\mathcal{A}$, then $s=r$, the rank of $\mathcal{A}$, and the Poincaré polynomial of the Orlik-Solomon algebra $A(\mathcal{A})$ of $\mathcal{A}$ factors into linear terms as follows:

$$
\operatorname{Poin}(A(\mathcal{A}), t)=\prod_{i=1}^{r}\left(1+\left|\pi_{i}\right| t\right),
$$

[Ter92] (cf. [OT92, Cor. 3.88]). Note that if $\mathcal{A}$ is free, then the Poincaré polynomial $\pi(\mathcal{A}, t)$ of $L(\mathcal{A})$ factors into linear terms as follows:

$$
\pi(\mathcal{A}, t)=\prod_{i=1}^{\ell}\left(1+b_{i} t\right),
$$

where $\exp \mathcal{A}=\left\{b_{1}, \ldots, b_{\ell}\right\}$ are the exponents of $\mathcal{A}$, [OT92, Thm. 4.137], see Theorem 2.5 below. It is natural to pose the question whether every nice arrangement is free, [Ter92]. This however is not the case; likewise, a free arrangement need not be factored in general, [Ter92].

In [HR16, Thm. 1.5], we gave an analogue of Terao's celebrated addition-deletion theorem for free arrangements for the class of nice arrangements, see Theorem 2.20 below.

Terao [Ter92] showed that every supersolvable arrangement is factored, see Proposition 2.18. Indeed, every supersolvable arrangement is inductively factored, see Proposition 2.24. Moreover, Jambu and Paris showed that each inductively factored arrangement is inductively free, see Proposition 2.26 ([JP95, Prop. 2.2]). Each of these classes of arrangements is properly contained in the other, see [HR16, Rem. 3.33].

Suppose that $W$ is a finite, unitary reflection group acting on the complex vector space $V$. Let $\mathcal{A}(W)=(\mathcal{A}(W), V)$ be the associated hyperplane arrangement of $W$. We refer to $\mathcal{A}(W)$ as a reflection arrangement. The aim of this paper is to classify all factored and all inductively factored reflection arrangements $\mathcal{A}(W)$.

In view of the aforementioned containments, we first recall the classifications of the inductively free and the supersolvable reflection arrangements, from [HR15, Thm. 1.1] and [HR14, Thm. 1.2], respectively. Here and later on we use the classification and labelling of the irreducible unitary reflection groups due to Shephard and Todd, [ST54].

Theorem 1.1. For $W$ a finite complex reflection group, the reflection arrangement $\mathcal{A}(W)$ of $W$ is inductively free if and only if $W$ does not admit an irreducible factor isomorphic to a monomial group $G(r, r, \ell)$ for $r, \ell \geqslant 3, G_{24}, G_{27}, G_{29}, G_{31}, G_{33}$, or $G_{34}$.

The case for Coxeter groups in Theorem 1.1 is due to Barakat and Cuntz [BC12]. 
Theorem 1.2. For $W$ a finite complex reflection group, $\mathcal{A}(W)$ is supersolvable if and only if any irreducible factor of $W$ is of rank at most 2 , is isomorphic either to a Coxeter group of type $A_{\ell}$ or $B_{\ell}$ for $\ell \geqslant 3$, or to a monomial group $G(r, p, \ell)$ for $r, \ell \geqslant 3$ and $p \neq r$.

We can now state our main classification results. Thanks to Proposition 2.29, the question whether $\mathcal{A}$ is nice reduces to the case when $\mathcal{A}$ is irreducible. Therefore, we may assume that $W$ is irreducible. In view of Theorem 1.2 and Proposition 2.24, we can state our classification results as follows:

Theorem 1.3. For $W$ a finite, irreducible, complex reflection group, $\mathcal{A}(W)$ is nice if and only if either $\mathcal{A}(W)$ is supersolvable or $W=G(r, r, 3)$ for $r \geqslant 3$.

Thanks to Proposition 2.30, the question whether $\mathcal{A}$ is inductively factored reduces to the case when $\mathcal{A}$ is irreducible. The classification of the inductively factored reflection arrangements is thus an immediate consequence of Theorems 1.1, 1.3 and Proposition 2.26 .

Corollary 1.4. For $W$ a finite, complex reflection group, $\mathcal{A}(W)$ is inductively factored if and only if it is supersolvable.

In contrast to Corollary 1.4, [HR16, Ex. 3.19] shows that among restrictions of reflection arrangements there are inductively factored instances which are not supersolvable.

A special case of a result due to Stanley implies that supersolvability of $\mathcal{A}$ is inherited by all restrictions $\mathcal{A}^{X}$, cf. [Sta72, Prop. 3.2]. While arbitrary inductively free arrangements are not hereditary, cf. [HR15, Ex. 2.16] and likewise for inductively factored arrangements, cf. [HR16, Ex. 3.28], for reflection arrangements, inductive freeness is hereditary, [HR15, Thm. 1.2]. Thus it is rather natural to ask whether the properties of being factored or inductively factored are also hereditary among reflection arrangements. These questions are answered in our next results:

Theorem 1.5. For $W$ a finite complex reflection group, $\mathcal{A}(W)$ is factored if and only if $\mathcal{A}(W)$ is hereditarily factored.

The following is an easy consequence of Corollary 1.4 and Stanley's result [Sta72, Prop. 3.2].

Corollary 1.6. For $W$ a finite complex reflection group, $\mathcal{A}(W)$ is inductively factored if and only if $\mathcal{A}(W)$ is hereditarily inductively factored.

The paper is organized as follows. Sections 2.1 and 2.2 recall basic notions and results for general hyperplane arrangements and their associated Orlik-Solomon algebras. Subsequently, we recall relevant concepts of free, inductively free and supersolvable arrangements in Sections 2.3 and 2.4. All this is taken from [OT92]. Sections 2.5 and 2.6 revisit the concepts and main results on nice and inductively factored arrangements from [Ter92], [JP95], and [HR16]. This is followed by a short recollection on hereditarily (inductively) factored arrangements in Section 2.7 from [HR16]. In Section 2.8, we discuss some required results on reflection arrangements. Finally, Theorems 1.3 and 1.5 are proved in Section 3.

For general information about arrangements and reflection groups we refer the reader to [OT92], [Bou68], [OS82] and [OT92, §4, §6]. 


\section{Recollections and Preliminaries}

\subsection{Hyperplane Arrangements}

Let $V=\mathbb{K}^{\ell}$ be an $\ell$-dimensional $\mathbb{K}$-vector space. A hyperplane arrangement is a pair $(\mathcal{A}, V)$, where $\mathcal{A}$ is a finite collection of hyperplanes in $V$. Usually, we simply write $\mathcal{A}$ in place of $(\mathcal{A}, V)$. We only consider central arrangements, i.e. $0 \in H$ for every $H \in \mathcal{A}$. We write $|\mathcal{A}|$ for the number of hyperplanes in $\mathcal{A}$. The empty arrangement in $V$ is denoted by $\Phi_{\ell}$.

The lattice $L(\mathcal{A})$ of $\mathcal{A}$ is the set of subspaces of $V$ of the form $H_{1} \cap \cdots \cap H_{r}$ where $\left\{H_{1}, \ldots, H_{r}\right\}$ is a subset of $\mathcal{A}$. For $X \in L(\mathcal{A})$, we have two associated arrangements, firstly the subarrangement $\mathcal{A}_{X}:=\{H \in \mathcal{A} \mid X \subseteq H\} \subseteq \mathcal{A}$ of $\mathcal{A}$ and secondly, the restriction of $\mathcal{A}$ to $X,\left(\mathcal{A}^{X}, X\right)$, where $\mathcal{A}^{X}:=\left\{X \cap H \mid H \in \mathcal{A} \backslash \mathcal{A}_{X}\right\}$. Note that $V$ belongs to $L(\mathcal{A})$ as the intersection of the empty collection of hyperplanes and $\mathcal{A}^{V}=\mathcal{A}$. The lattice $L(\mathcal{A})$ is a partially ordered set by reverse inclusion: $X \leqslant Y$ provided $Y \subseteq X$ for $X, Y \in L(\mathcal{A})$. We have a $r a n k$ function on $L(\mathcal{A}): r(X):=\operatorname{codim}_{V}(X)$. The $\operatorname{rank} r(\mathcal{A})$ of $\mathcal{A}$ is the rank of a maximal element in $L(\mathcal{A})$ with respect to the partial order. With this definition $L(\mathcal{A})$ is a geometric lattice, [OT92, p. 24]. Let $T_{\mathcal{A}}=\cap_{H \in \mathcal{A}} H$ be the center of $L(\mathcal{A})$. If $\mathcal{A}$ is central, then $0 \in T_{\mathcal{A}}$. The $\ell$-arrangement $\mathcal{A}$ is called essential provided $r(\mathcal{A})=\ell$. If $\mathcal{A}$ is essential and central, then $T_{\mathcal{A}}=\{0\}$.

Let $\mathcal{A}$ be central and let $X, Y \in L(\mathcal{A})$ with $X<Y$. We recall the following sublattices of $L(\mathcal{A})$ from [OT92, Def. 2.10], $L(\mathcal{A})_{Y}:=\{Z \in L(\mathcal{A}) \mid Z \leqslant Y\}, L(\mathcal{A})^{X}:=\{Z \in L(\mathcal{A}) \mid$ $X \leqslant Z\}$, and the interval $[X, Y]:=L(\mathcal{A})_{Y} \cap L(\mathcal{A})^{X}=\{Z \in L(\mathcal{A}) \mid X \leqslant Z \leqslant Y\}$.

For $\mathcal{A} \neq \Phi_{\ell}$, let $H_{0} \in \mathcal{A}$. Define $\mathcal{A}^{\prime}:=\mathcal{A} \backslash\left\{H_{0}\right\}$, and $\mathcal{A}^{\prime \prime}:=\mathcal{A}^{H_{0}}=\left\{H_{0} \cap H \mid H \in \mathcal{A}^{\prime}\right\}$. Then $\left(\mathcal{A}, \mathcal{A}^{\prime}, \mathcal{A}^{\prime \prime}\right)$ is a triple of arrangements, [OT92, Def. 1.14]. by

The product $\mathcal{A}=\left(\mathcal{A}_{1} \times \mathcal{A}_{2}, V_{1} \oplus V_{2}\right)$ of two arrangements $\left(\mathcal{A}_{1}, V_{1}\right),\left(\mathcal{A}_{2}, V_{2}\right)$ is defined

$$
\mathcal{A}=\mathcal{A}_{1} \times \mathcal{A}_{2}:=\left\{H_{1} \oplus V_{2} \mid H_{1} \in \mathcal{A}_{1}\right\} \cup\left\{V_{1} \oplus H_{2} \mid H_{2} \in \mathcal{A}_{2}\right\},
$$

see [OT92, Def. 2.13].

Note that $\mathcal{A} \times \Phi_{0}=\mathcal{A}$ for any arrangement $\mathcal{A}$. If $\mathcal{A}$ is of the form $\mathcal{A}=\mathcal{A}_{1} \times \mathcal{A}_{2}$, where $\mathcal{A}_{i} \neq \Phi_{0}$ for $i=1,2$, then $\mathcal{A}$ is called reducible, else $\mathcal{A}$ is irreducible, [OT92, Def. 2.15].

\subsection{The Orlik-Solomon Algebra of an Arrangement}

The most basic algebraic invariant associated with an arrangement $\mathcal{A}$ is its so called Orlik-Solomon algebra $A(\mathcal{A})$, introduced by Orlik and Solomon in [OS80]. The $\mathbb{K}$-algebra $A(\mathcal{A})$ is a graded and anti-commutative. It is generated by 1 in degree 0 and by a set of degree 1 generators $\left\{a_{H} \mid H \in \mathcal{A}\right\}$, e.g. see [OT92, §3.1]. Let $A(\mathcal{A})=\oplus_{i=0}^{r} A(\mathcal{A})_{i}$ be the decomposition of $A(\mathcal{A})$ into homogeneous components, so that $\operatorname{Poin}(A(\mathcal{A}), t)=$ $\sum_{i=0}^{r}\left(\operatorname{dim} A(\mathcal{A})_{i}\right) t^{i}$, where $r=r(\mathcal{A})$ is the rank of $\mathcal{A}$. In particular, $A(\mathcal{A})_{0}=\mathbb{K}$ and $A(\mathcal{A})_{1}=\sum_{H \in \mathcal{A}} \mathbb{K} a_{H}$.

Thanks to a fundamental result due to Orlik and Solomon [OS80, Thm. 2.6] (cf. [OT92, Thm. 3.68]), the Poincaré polynomial of $A(\mathcal{A})$ coincides with the combinatorially defined 
Poincaré polynomial $\pi(\mathcal{A}, t)$ of $\mathcal{A}$,

$$
\operatorname{Poin}(A(\mathcal{A}), t)=\pi(\mathcal{A}, t) .
$$

The geometric significance of $A(\mathcal{A})$ stems from the fact that in case $\mathbb{K}=\mathbb{C}$ is the field of complex numbers, Orlik and Solomon showed in [OS80, Thm. 5.2] that as an associative, graded $\mathbb{C}$-algebra $A(\mathcal{A})$ is isomorphic to the cohomology algebra of the complement $M(\mathcal{A})$ of the complex arrangement $\mathcal{A}$ (cf. [OT92, §5.4]):

$$
A(\mathcal{A}) \cong H^{*}(M(\mathcal{A})) .
$$

In particular, the Poincaré polynomial $\operatorname{Poin}(M(\mathcal{A}), t)$ of $M(\mathcal{A})$ is given by $\operatorname{Poin}(A(\mathcal{A}), t)$. Let $\pi=\left(\pi_{1}, \ldots, \pi_{s}\right)$ be a partition of $\mathcal{A}$ and let

$$
\left[\pi_{i}\right]:=\mathbb{K}+\sum_{H \in \pi_{i}} \mathbb{K} a_{H}
$$

be the $\mathbb{K}$-subspace of $A(\mathcal{A})$ spanned by 1 and the set of $\mathbb{K}$-algebra generators $a_{H}$ of $A(\mathcal{A})$ corresponding to the members in $\pi_{i}$. So the Poincaré polynomial of the graded $\mathbb{K}$-vector space $\left[\pi_{i}\right]$ is just $\operatorname{Poin}\left(\left[\pi_{i}\right], t\right)=1+\left|\pi_{i}\right| t$. Consider the canonical $\mathbb{K}$-linear map

$$
\kappa:\left[\pi_{1}\right] \otimes \cdots \otimes\left[\pi_{s}\right] \rightarrow A(\mathcal{A})
$$

given by multiplication. We say that $\pi$ gives rise to a tensor factorization of $A(\mathcal{A})$ if $\kappa$ is an isomorphism of graded $\mathbb{K}$-vector spaces. In this case $s=r$, as $r$ is the top degree of $A(\mathcal{A})$, and thus we get a factorization of the Poincaré polynomial of $A(\mathcal{A})$ into linear terms

$$
\operatorname{Poin}(A(\mathcal{A}), t)=\prod_{i=1}^{r}\left(1+\left|\pi_{i}\right| t\right) .
$$

For $\mathcal{A}=\Phi_{\ell}$ the empty arrangement, we set $[\varnothing]:=\mathbb{K}$, so that $\kappa:[\varnothing] \cong A\left(\Phi_{\ell}\right)$.

In [OST84, Thm. 5.3], Orlik, Solomon and Terao showed that a supersolvable arrangement $\mathcal{A}$ admits a partition $\pi$ which gives rise to a tensor factorization of $A(\mathcal{A})$ via $\kappa$ in (2.2) (cf. [OT92, Thm. 3.81]); see Proposition 2.18 below.

In [Ter92, Thm. 2.8], Terao proved that $\pi$ gives rise to a tensor factorization of the Orlik-Solomon algebra $A(\mathcal{A})$ via $\kappa$ as in (2.2) if and only if $\pi$ is nice for $\mathcal{A}$ (Definition 2.12), see Theorem 2.14 (cf. [OT92, Thm. 3.87]). So nice arrangements are a generalization of supersolvable ones. Note that $\kappa$ is not an isomorphism of $\mathbb{K}$-algebras.

\subsection{Free and inductively free Arrangements}

Free arrangements play a crucial role in the theory of arrangements. See [OT92, §4] for the definition and basic properties of free arrangements. If $\mathcal{A}$ is free, then we can associate with $\mathcal{A}$ the multiset of its exponents, denoted $\exp \mathcal{A}$.

Owing to [OT92, Prop. 4.28], free arrangements behave well with respect to the product construction for arrangements. 
Proposition 2.3. Let $\mathcal{A}_{1}, \mathcal{A}_{2}$ be two arrangements. Then $\mathcal{A}=\mathcal{A}_{1} \times \mathcal{A}_{2}$ is free if and only if both $\mathcal{A}_{1}$ and $\mathcal{A}_{2}$ are free and in that case the multiset of exponents of $\mathcal{A}$ is given by $\exp \mathcal{A}=\left\{\exp \mathcal{A}_{1}, \exp \mathcal{A}_{2}\right\}$.

Terao's celebrated Addition-Deletion Theorem [Ter80] plays a pivotal role in the study of free arrangements, [OT92, Thm. 4.51].

Theorem 2.4. Suppose that $\mathcal{A} \neq \Phi_{\ell}$. Let $\left(\mathcal{A}, \mathcal{A}^{\prime}, \mathcal{A}^{\prime \prime}\right)$ be a triple of arrangements. Then any two of the following statements imply the third:

(i) $\mathcal{A}$ is free with $\exp \mathcal{A}=\left\{b_{1}, \ldots, b_{\ell-1}, b_{\ell}\right\}$;

(ii) $\mathcal{A}^{\prime}$ is free with $\exp \mathcal{A}^{\prime}=\left\{b_{1}, \ldots, b_{\ell-1}, b_{\ell}-1\right\}$;

(iii) $\mathcal{A}^{\prime \prime}$ is free with $\exp \mathcal{A}^{\prime \prime}=\left\{b_{1}, \ldots, b_{\ell-1}\right\}$.

Terao's Factorization Theorem [Ter81] shows that the Poincaré polynomial $\pi(\mathcal{A}, t)$ of a free arrangement $\mathcal{A}$ factors into linear terms given by the exponents of $\mathcal{A}$ (cf. [OT92, Thm. 4.137]):

Theorem 2.5. Suppose that $\mathcal{A}$ is free with $\exp \mathcal{A}=\left\{b_{1}, \ldots, b_{\ell}\right\}$. Then

$$
\pi(\mathcal{A}, t)=\prod_{i=1}^{\ell}\left(1+b_{i} t\right) .
$$

Theorem 2.4 motivates the notion of inductively free arrangements, [OT92, Def. 4.53].

Definition 2.6. The class $\mathcal{I F}$ of inductively free arrangements is the smallest class of arrangements subject to

(i) $\Phi_{\ell} \in \mathcal{I F}$ for each $\ell \geqslant 0$;

(ii) if there exists a hyperplane $H_{0} \in \mathcal{A}$ such that both $\mathcal{A}^{\prime}$ and $\mathcal{A}^{\prime \prime}$ belong to $\mathcal{I} \mathcal{F}$, and $\exp \mathcal{A}^{\prime \prime} \subseteq \exp \mathcal{A}^{\prime}$, then $\mathcal{A}$ also belongs to $\mathcal{I} \mathcal{F}$.

In [HR15, Prop. 2.10], we showed that the compatibility of products with free arrangements from Proposition 2.3 restricts to inductively free arrangements.

Proposition 2.7. Let $\mathcal{A}_{1}, \mathcal{A}_{2}$ be two arrangements. Then $\mathcal{A}=\mathcal{A}_{1} \times \mathcal{A}_{2}$ is inductively free if and only if both $\mathcal{A}_{1}$ and $\mathcal{A}_{2}$ are inductively free and in that case $\exp \mathcal{A}=$ $\left\{\exp \mathcal{A}_{1}, \exp \mathcal{A}_{2}\right\}$.

\subsection{Supersolvable Arrangements}

Let $\mathcal{A}$ be an arrangement. Following [OT92, §2], we say that $X \in L(\mathcal{A})$ is modular provided $X+Y \in L(\mathcal{A})$ for every $Y \in L(\mathcal{A})$, cf. [OT92, Def. 2.32, Cor. 2.26].

The following notion is due to Stanley [Sta72]. 
Definition 2.8. Let $\mathcal{A}$ be a central (and essential) $\ell$-arrangement. We say that $\mathcal{A}$ is supersolvable provided there is a maximal chain

$$
V=X_{0}<X_{1}<\ldots<X_{\ell-1}<X_{\ell}=\{0\}
$$

of modular elements $X_{i}$ in $L(\mathcal{A})$.

Remark 2.9. By [OT92, Ex. 2.28], $V,\{0\}$ and the members in $\mathcal{A}$ are always modular in $L(\mathcal{A})$. It follows that all $0-1-$, and 2-arrangements are supersolvable.

Note that supersolvable arrangements are always inductively free, [OT92, Thm. 4.58].

In [HR14, Prop. 2.6], we showed that the compatibility of products with inductively free arrangements from Proposition 2.7 restricts further to supersolvable arrangements.

\subsection{Nice Arrangements}

The notion of a nice or factored arrangement goes back to Terao [Ter92]. It generalizes the concept of a supersolvable arrangement, see Proposition 2.18. We recall the relevant notions and results from [Ter92] (cf. [OT92, §2.3]).

Definition 2.10. Let $\pi=\left(\pi_{1}, \ldots, \pi_{s}\right)$ be a partition of $\mathcal{A}$. Then $\pi$ is called independent, provided for any choice $H_{i} \in \pi_{i}$ for $1 \leqslant i \leqslant s$, the resulting $s$ hyperplanes are linearly independent, i.e. $r\left(H_{1} \cap \ldots \cap H_{s}\right)=s$.

Definition 2.11. Let $\pi=\left(\pi_{1}, \ldots, \pi_{s}\right)$ be a partition of $\mathcal{A}$ and let $X \in L(\mathcal{A})$. The induced partition $\pi_{X}$ of $\mathcal{A}_{X}$ is given by the non-empty blocks of the form $\pi_{i} \cap \mathcal{A}_{X}$.

Definition 2.12. The partition $\pi$ of $\mathcal{A}$ is nice for $\mathcal{A}$ or a factorization of $\mathcal{A}$ provided

(i) $\pi$ is independent, and

(ii) for each $X \in L(\mathcal{A}) \backslash\{V\}$, the induced partition $\pi_{X}$ admits a block which is a singleton.

If $\mathcal{A}$ admits a factorization, then we also say that $\mathcal{A}$ is factored or nice.

Remark 2.13. (i). Vacuously, the empty partition is nice for the empty arrangement $\Phi_{\ell}$.

(ii). If $\mathcal{A} \neq \Phi_{\ell}, \pi$ is a nice partition of $\mathcal{A}$ and $X \in L(\mathcal{A}) \backslash\{V\}$, then the non-empty parts of the induced partition $\pi_{X}$ form a nice partition of $\mathcal{A}_{X}$; cf. the proof of [Ter92, Cor. 2.11].

(iii). Since the singleton condition in Definition 2.12(ii) also applies to the center $T_{\mathcal{A}}$ of $L(\mathcal{A})$, a factorization $\pi$ of $\mathcal{A} \neq \Phi_{\ell}$ always admits a singleton as one of its parts. Also note that for a hyperplane, the singleton condition trivially holds.

We recall the main results from [Ter92] (cf. [OT92, §3.3]) that motivated Definition 2.12 . 
Theorem 2.14. Let $\mathcal{A}$ be a central $\ell$-arrangement and let $\pi=\left(\pi_{1}, \ldots, \pi_{s}\right)$ be a partition of $\mathcal{A}$. Then the $\mathbb{K}$-linear map $\kappa$ defined in (2.2) is an isomorphism of graded $\mathbb{K}$-vector spaces if and only if $\pi$ is nice for $\mathcal{A}$.

Corollary 2.15. Let $\pi=\left(\pi_{1}, \ldots, \pi_{s}\right)$ be a factorization of $\mathcal{A}$. Then the following hold:

(i) $s=r=r(\mathcal{A})$ and

$$
\operatorname{Poin}(A(\mathcal{A}), t)=\prod_{i=1}^{r}\left(1+\left|\pi_{i}\right| t\right) ;
$$

(ii) the multiset $\left\{\left|\pi_{1}\right|, \ldots,\left|\pi_{r}\right|\right\}$ only depends on $\mathcal{A}$;

(iii) for any $X \in L(\mathcal{A})$, we have

$$
r(X)=\left|\left\{i \mid \pi_{i} \cap \mathcal{A}_{X} \neq \varnothing\right\}\right|
$$

Remark 2.16. It follows from (2.1) and Corollary 2.15 that the question whether $\mathcal{A}$ is factored is a purely combinatorial property and only depends on the lattice $L(\mathcal{A})$.

Remark 2.17. Suppose that $\mathcal{A}$ is free of rank $r$. Then $\mathcal{A}=\Phi_{\ell-r} \times \mathcal{A}_{0}$, where $\mathcal{A}_{0}$ is an essential, free $r$-arrangement (cf. [OT92, §3.2]), and so, thanks to Proposition 2.3, $\exp \mathcal{A}=$ $\left\{0^{\ell-r}, \exp \mathcal{A}_{0}\right\}$. Suppose that $\pi=\left(\pi_{1}, \ldots, \pi_{r}\right)$ is a nice partition of $\mathcal{A}$. Then by the factorization properties of the Poincaré polynomials for free and factored arrangements, Theorem 2.5, respectively Corollary 2.15(i) and (2.1) we have

$$
\exp \mathcal{A}=\left\{0^{\ell-r},\left|\pi_{1}\right|, \ldots,\left|\pi_{r}\right|\right\} .
$$

In particular, if $\mathcal{A}$ is essential, then

$$
\exp \mathcal{A}=\left\{\left|\pi_{1}\right|, \ldots,\left|\pi_{\ell}\right|\right\}
$$

Finally, we record [Ter92, Ex. 2.4], which shows that nice arrangements generalize supersolvable ones (cf. [OST84, Thm. 5.3], [J90, Prop. 3.2.2], [OT92, Prop. 2.67, Thm. $3.81])$.

Proposition 2.18. Let $\mathcal{A}$ be a central, supersolvable arrangement of rank $r$. Let

$$
V=X_{0}<X_{1}<\ldots<X_{r-1}<X_{r}=T_{\mathcal{A}}
$$

be a maximal chain of modular elements in $L(\mathcal{A})$. Define $\pi_{i}=\mathcal{A}_{X_{i}} \backslash \mathcal{A}_{X_{i-1}}$ for $1 \leqslant i \leqslant r$. Then $\pi=\left(\pi_{1}, \ldots, \pi_{r}\right)$ is a nice partition of $\mathcal{A}$. In particular, the $\mathbb{K}$-linear map $\kappa$ defined in (2.2) is an isomorphism of graded $\mathbb{K}$-vector spaces.

\subsection{Inductively factored Arrangements}

Following Jambu and Paris [JP95], we introduce further notation. 
Definition 2.19. Suppose $\mathcal{A} \neq \Phi_{\ell}$. Let $\pi=\left(\pi_{1}, \ldots, \pi_{s}\right)$ be a partition of $\mathcal{A}$. Let $H_{0} \in \pi_{1}$ and let $\left(\mathcal{A}, \mathcal{A}^{\prime}, \mathcal{A}^{\prime \prime}\right)$ be the triple associated with $H_{0}$. We say that $H_{0}$ is distinguished (with respect to $\pi$ ) provided $\pi$ induces a factorization $\pi^{\prime}$ of $\mathcal{A}^{\prime}$, i.e. the non-empty subsets $\pi_{i} \cap \mathcal{A}^{\prime}$ form a nice partition of $\mathcal{A}^{\prime}$. Note that since $H_{0} \in \pi_{1}$, we have $\pi_{i} \cap \mathcal{A}^{\prime}=\pi_{i} \neq \varnothing$ for $i=2, \ldots, s$.

Also, associated with $\pi$ and $H_{0}$, we define the restriction map

$$
\varrho:=\varrho_{\pi, H_{0}}: \mathcal{A} \backslash \pi_{1} \rightarrow \mathcal{A}^{\prime \prime} \text { given by } H \mapsto H \cap H_{0}
$$

and set

$$
\pi_{i}^{\prime \prime}:=\varrho\left(\pi_{i}\right)=\left\{H \cap H_{0} \mid H \in \pi_{i}\right\} \text { for } 2 \leqslant i \leqslant s .
$$

In general $\varrho$ need not be surjective nor injective. However, since we are only concerned with cases when $\pi^{\prime \prime}=\left(\pi_{2}^{\prime \prime}, \ldots, \pi_{s}^{\prime}\right)$ is a partition of $\mathcal{A}^{\prime \prime}, \varrho$ has to be onto and $\varrho\left(\pi_{i}\right) \cap \varrho\left(\pi_{j}\right)=$ $\varnothing$ for $i \neq j$. As we have observed in [HR16], the crucial condition in this context is the injectivity of $\varrho$.

In [HR16, Thm. 1.5, Thm. 1.7], we gave the following analogues of Terao's AdditionDeletion Theorem 2.4 for free arrangements for the class of nice arrangements.

Theorem 2.20. Suppose that $\mathcal{A} \neq \Phi_{\ell}$. Let $\pi=\left(\pi_{1}, \ldots, \pi_{\ell}\right)$ be a partition of $\mathcal{A}$. Let $H_{0} \in \pi_{1}$ and let $\left(\mathcal{A}, \mathcal{A}^{\prime}, \mathcal{A}^{\prime \prime}\right)$ be the triple associated with $H_{0}$. Suppose that $\varrho: \mathcal{A} \backslash \pi_{1} \rightarrow \mathcal{A}^{\prime \prime}$ is bijective. Then any two of the following statements imply the third:

(i) $\pi$ is nice for $\mathcal{A}$;

(ii) $\pi^{\prime}$ is nice for $\mathcal{A}^{\prime}$;

(iii) $\pi^{\prime \prime}$ is nice for $\mathcal{A}^{\prime \prime}$.

As indicated above, nice arrangements need not be free and vice versa, as observed by Terao [Ter92]. Combining Theorem 2.20 with Terao's Addition-Deletion Theorem 2.4 for free arrangements, in [HR16, Thm. 1.7] we obtain the following Addition-Deletion Theorem for the proper subclass of free and nice arrangements.

Theorem 2.21. Suppose that $\mathcal{A} \neq \Phi_{\ell}$. Let $\pi=\left(\pi_{1}, \ldots, \pi_{\ell}\right)$ be a partition of $\mathcal{A}$. Let $H_{0} \in \pi_{\ell}$ and let $\left(\mathcal{A}, \mathcal{A}^{\prime}, \mathcal{A}^{\prime \prime}\right)$ be the triple associated with $H_{0}$. Suppose that $\varrho: \mathcal{A} \backslash \pi_{1} \rightarrow \mathcal{A}^{\prime \prime}$ is bijective. Then any two of the following statements imply the third:

(i) $\pi$ is nice for $\mathcal{A}$ and $\mathcal{A}$ is free;

(ii) $\pi^{\prime}$ is nice for $\mathcal{A}^{\prime}$ and $\mathcal{A}^{\prime}$ is free;

(iii) $\pi^{\prime \prime}$ is nice for $\mathcal{A}^{\prime \prime}$ and $\mathcal{A}^{\prime \prime}$ is free.

Worth noting is the fact that in Theorem 2.21 we do not need to explicitly require the containment conditions on the sets of exponents of the arrangements involved, see Theorem 2.4. This is a consequence of the presence of the underlying factorizations. 
We also note that the bijectivity condition on $\varrho$ in both theorems is necessary, see [HR16, Ex. 3.3, Ex. 3.20].

The Addition-Deletion Theorem 2.20 for nice arrangements motivates the following stronger notion of factorization, cf. [JP95], [HR16, Def. 3.8].

Definition 2.22. The class $\mathcal{I} \mathcal{F} \mathcal{A C}$ of inductively factored arrangements is the smallest class of pairs $(\mathcal{A}, \pi)$ of arrangements $\mathcal{A}$ together with a partition $\pi$ subject to

(i) $\left(\Phi_{\ell},(\varnothing)\right) \in \mathcal{I} \mathcal{F} \mathcal{A C}$ for each $\ell \geqslant 0$;

(ii) if there exists a partition $\pi$ of $\mathcal{A}$ and a hyperplane $H_{0} \in \pi_{1}$ such that for the triple $\left(\mathcal{A}, \mathcal{A}^{\prime}, \mathcal{A}^{\prime \prime}\right)$ associated with $H_{0}$ the restriction map $\varrho=\varrho_{\pi, H_{0}}: \mathcal{A} \backslash \pi_{1} \rightarrow \mathcal{A}^{\prime \prime}$ is bijective and for the induced partitions $\pi^{\prime}$ of $\mathcal{A}^{\prime}$ and $\pi^{\prime \prime}$ of $\mathcal{A}^{\prime \prime}$ both $\left(\mathcal{A}^{\prime}, \pi^{\prime}\right)$ and $\left(\mathcal{A}^{\prime \prime}, \pi^{\prime \prime}\right)$ belong to $\mathcal{I} \mathcal{F} \mathcal{A C}$, then $(\mathcal{A}, \pi)$ also belongs to $\mathcal{I} \mathcal{F} \mathcal{A C}$.

If $(\mathcal{A}, \pi)$ is in $\mathcal{I} \mathcal{F} \mathcal{A C}$, then we say that $\mathcal{A}$ is inductively factored with respect to $\pi$, or else that $\pi$ is an inductive factorization of $\mathcal{A}$. Sometimes, we simply say $\mathcal{A}$ is inductively factored without reference to a specific inductive factorization of $\mathcal{A}$.

Our definition of inductively factored arrangements in Definition 2.22 differs slightly from the one by Jambu and Paris [JP95]; see [HR16, Rem. 3.9].

Definition 2.23. In analogy to hereditary freeness and hereditary inductive freeness, [OT92, Def. 4.140, p. 253], we say that $\mathcal{A}$ is hereditarily factored provided $\mathcal{A}^{X}$ is factored for every $X \in L(\mathcal{A})$ and that $\mathcal{A}$ is hereditarily inductively factored provided $\mathcal{A}^{X}$ is inductively factored for every $X \in L(\mathcal{A})$.

In [HR16, Prop. 3.11], we strengthened Proposition 2.18 as follows.

Proposition 2.24. If $\mathcal{A}$ is supersolvable, then $\mathcal{A}$ is inductively factored.

Remark 2.25. Since any 1- and 2-arrangement is supersolvable, Remark 2.9, each such is inductively factored, by Proposition 2.24.

In [JP95, Prop. 2.2], Jambu and Paris showed that inductively factored arrangements are always inductively free; see also [HR16, Prop. 3.14]. (Jambu and Paris only claimed freeness but their proof actually does give the stronger result.)

Proposition 2.26. Let $\pi=\left(\pi_{1}, \ldots, \pi_{r}\right)$ be an inductive factorization of $\mathcal{A}$. Then $\mathcal{A}$ is inductively free with exponents $\exp \mathcal{A}=\left\{0^{\ell-r},\left|\pi_{1}\right|, \ldots,\left|\pi_{r}\right|\right\}$.

Remark 2.27. The converse of Proposition 2.26 is false, Terao has already noted that the reflection arrangement $\mathcal{A}\left(D_{4}\right)$ of the Coxeter group of type $D_{4}$ is not factored. But $\mathcal{A}\left(D_{4}\right)$ is inductively free, [JT84, Ex. 2.6].

Remark 2.28. Jambu and Paris observed that the reflection arrangement $\mathcal{A}(G(3,3,3))$ of the complex reflection group $G(3,3,3)$ is factored but not inductively factored [JP95]. Note that $\mathcal{A}(G(3,3,3))$ is not inductively free, [HR15, Thm. 1.1]. In particular, a free and factored arrangement need not be inductively factored; see also [HR16, Ex. 3.20]. Not even an inductively free and factored arrangement need be inductively factored, see [HR16, Ex. 3.22]. 
In [HR16, Prop. 3.29], we showed that the product construction behaves well with factorizations.

Proposition 2.29. Let $\mathcal{A}_{1}, \mathcal{A}_{2}$ be two arrangements. Then $\mathcal{A}=\mathcal{A}_{1} \times \mathcal{A}_{2}$ is nice if and only if both $\mathcal{A}_{1}$ and $\mathcal{A}_{2}$ are nice.

And in [HR16, Prop. 3.30], we strengthened Proposition 2.29 further by showing that the compatibility with products restricts to the class of inductively factored arrangements.

Proposition 2.30. Let $\mathcal{A}_{1}, \mathcal{A}_{2}$ be two arrangements. Then $\mathcal{A}=\mathcal{A}_{1} \times \mathcal{A}_{2}$ is inductively factored if and only if both $\mathcal{A}_{1}$ and $\mathcal{A}_{2}$ are inductively factored and in that case the multiset of exponents of $\mathcal{A}$ is given by $\exp \mathcal{A}=\left\{\exp \mathcal{A}_{1}, \exp \mathcal{A}_{2}\right\}$.

\subsection{Hereditarily factored Arrangements}

While a 2-arrangement is always inductively factored, by Remark 2.9, in general, a factored 3-arrangement need not be inductively factored, not even if it is a reflection arrangement, see Remark 2.28. Nevertheless, for a 3-arrangement, we have the following counterpart to [HR15, Lem. 2.15] in our setting; see [HR16, Lem. 3.27].

Lemma 2.31. Suppose that $\ell=3$. Then $\mathcal{A}$ is (inductively) factored if and only if it is hereditarily (inductively) factored.

The compatibility from Propositions 2.29 and 2.30 restricts further to the classes of hereditarily factored and hereditarily inductively factored arrangements, respectively; see [HR16, Cor. 3.32].

Corollary 2.32. Let $\mathcal{A}_{1}, \mathcal{A}_{2}$ be two arrangements. Then $\mathcal{A}=\mathcal{A}_{1} \times \mathcal{A}_{2}$ is hereditarily (inductively) factored if and only if both $\mathcal{A}_{1}$ and $\mathcal{A}_{2}$ are hereditarily (inductively) factored. In case of inductively factored arrangements the multiset of exponents of $\mathcal{A}$ is given by $\exp \mathcal{A}=\left\{\exp \mathcal{A}_{1}, \exp \mathcal{A}_{2}\right\}$

\subsection{Reflection Groups and Reflection Arrangements}

The irreducible finite complex reflection groups were classified by Shephard and Todd, [ST54]. Let $W \subseteq \mathrm{GL}(V)$ be a finite complex reflection group. For $w \in W$, we write $\operatorname{Fix}(w):=\{v \in V \mid w v=v\}$ for the fixed point subspace of $w$. For $U \subseteq V$ a subspace, we define the parabolic subgroup $W_{U}$ of $W$ by $W_{U}:=\{w \in W \mid U \subseteq \operatorname{Fix}(w)\}$.

The reflection arrangement $\mathcal{A}=\mathcal{A}(W)$ of $W$ in $V$ is the hyperplane arrangement consisting of the reflecting hyperplanes of the elements in $W$ acting as reflections on $V$. By Steinberg's Theorem [Ste60, Thm. 1.5], for $U \subseteq V$ a subspace, the parabolic subgroup $W_{U}$ is itself a complex reflection group, generated by the unitary reflections in $W$ that are contained in $W_{U}$. Thus, we identify the reflection arrangement $\mathcal{A}\left(W_{U}\right)$ of $W_{U}$ as a subarrangement of $\mathcal{A}$.

First we record a consequence of the classification of the inductively free reflection arrangements, Theorem 1.1: 
Corollary 2.33. Let $\mathcal{A}=\mathcal{A}(W)$ be an inductively free reflection arrangement. Then $\mathcal{A}\left(W_{X}\right)$ is also inductively free for any parabolic subgroup $W_{X}$ of $W$.

Proof. The result follows readily from Theorem 1.1 and the classification of the parabolic subgroups of the irreducible complex reflection groups, [OT92, §6.4, App. C].

Next we record an elementary but nevertheless very useful inductive tool.

Lemma 2.34. Let $\mathcal{A}=\mathcal{A}(W)$ be a nice reflection arrangement. Then $\mathcal{A}\left(W_{X}\right)$ is also nice for any parabolic subgroup $W_{X}$ of $W$.

Proof. Note that for $X \in L(\mathcal{A})$, we have $\mathcal{A}\left(W_{X}\right)=\mathcal{A}_{X}$, cf. [OT92, Thm. 6.27, Cor. 6.28]. The desired result follows from Remark 2.13(ii).

Using Corollary 1.4, Lemma 2.34 restricts to inductively factored reflection arrangements:

Corollary 2.35. Let $\mathcal{A}=\mathcal{A}(W)$ be an inductively factored reflection arrangement. Then $\mathcal{A}\left(W_{X}\right)$ is also inductively factored for any parabolic subgroup $W_{X}$ of $W$.

Proof. As noted above, for $X \in L(\mathcal{A})$, we have $\mathcal{A}\left(W_{X}\right)=\mathcal{A}_{X}$, cf. [OT92, Thm. 6.27, Cor. 6.28]. Moreover, since $L\left(\mathcal{A}_{X}\right)=L(\mathcal{A})_{X}=[V, X]$ is an interval in $L(\mathcal{A})$, [OT92, Def. 2.10], the result follows from Corollary 1.4 and Stanley's result [Sta72, Prop. 3.2].

\section{Nice Reflection Arrangements}

In this section we provide proofs of Theorems 1.3 and 1.5.

\subsection{Proof of Theorem 1.3.}

It follows from Proposition 2.18 that if $\mathcal{A}$ is supersolvable, then it is nice. Thus, by Proposition 2.29, we may assume that $W$ is irreducible so that $\mathcal{A}(W)$ is not supersolvable. Then in view of Theorem 1.2, Theorem 1.3 follows from Lemmas $3.1-3.10$ below.

Lemma 3.1. Let $W=G(r, r, 3)$ for $r \geqslant 3$. Then $\mathcal{A}(W)$ is nice.

Proof. Let $r \geqslant 3, W=G(r, r, 3)$ and $\mathcal{A}=\mathcal{A}(W)$. We denote the coordinate functions of $\mathbb{C}^{3}$ by $x, y$ and $z$. Furthermore, let $\zeta$ be a primitive $r$-th root of 1 . There are three families of hyperplanes in $\mathcal{A}$ given as follows. For $i=0, \ldots, r-1$, we set

$$
A_{i}:=\operatorname{ker}\left(x-\zeta^{i} y\right), B_{i}:=\operatorname{ker}\left(x-\zeta^{i} z\right) \text {, and } \quad C_{i}=\operatorname{ker}\left(y-\zeta^{i} z\right) .
$$

We also consider the hyperplanes $A_{i}, B_{i}$, and $C_{i}$ for $i \in \mathbb{Z}$, simply by taking $i$ modulo $r$.

One readily checks that the members $X$ of rank 2 of $L(\mathcal{A})$ are given by the following subarrangements $\mathcal{A}_{X}$ of $\mathcal{A}$ (i.e. $X=\cap_{H \in \mathcal{A}_{X}} H$ ) :

$$
\left\{A_{0}, A_{1}, \ldots, A_{r-1}\right\},\left\{B_{0}, B_{1}, \ldots, B_{r-1}\right\},\left\{C_{0}, C_{1}, \ldots, C_{r-1}\right\},
$$


and

$$
\left\{A_{i}, B_{j}, C_{k} \mid i, j \in\{0, \ldots, r-1\} \text { and } k=j-i \bmod r\right\} .
$$

Note that $\exp \mathcal{A}(G(r, r, 3))=\{1, r+1,2(r-1)\}$, cf. [OT92, Cor. 6.86]. We claim that the following partition $\pi$ is a factorization of $\mathcal{A}$ :

$$
\pi=\left(\pi_{1}, \pi_{2}, \pi_{3}\right):=\left(\left\{A_{0}\right\},\left\{A_{1}, \ldots, A_{r-1}, B_{0}, C_{0}\right\},\left\{B_{1}, \ldots, B_{r-1}, C_{1}, \ldots, C_{r-1}\right\}\right) .
$$

First we check the singleton condition from Definition 2.12(ii). Thanks to Remark 2.13(iii), we only need to check this for $X$ of rank 2 .

If $\mathcal{A}_{X}=\left\{A_{0}, A_{1}, \ldots, A_{r-1}\right\}$, then $\pi_{1} \cap \mathcal{A}_{X}=\left\{A_{0}\right\}$. Likewise, if $\mathcal{A}_{X}=\left\{B_{0}, \ldots, B_{r-1}\right\}$, respectively $\mathcal{A}_{X}=\left\{C_{0}, C_{1}, \ldots, C_{r-1}\right\}$, then $\pi_{2} \cap \mathcal{A}_{X}=\left\{B_{0}\right\}$, respectively $\pi_{2} \cap \mathcal{A}_{X}=\left\{C_{0}\right\}$, is the desired singleton. If $\mathcal{A}_{X}=\left\{A_{0}, B_{j}, C_{0-j}\right\}$, then $\pi_{1} \cap \mathcal{A}_{X}=\left\{A_{0}\right\}$. Finally, let $\mathcal{A}_{X}=\left\{A_{i}, B_{j}, C_{i-j}\right\}$ for $i, j \neq 0 \bmod r$. If $i \neq j$, then $\pi_{2} \cap \mathcal{A}_{X}=\left\{A_{i}\right\}$, else if $i=j$, then $\pi_{3} \cap \mathcal{A}_{X}=\left\{B_{j}\right\}$, and if $\mathcal{A}_{X}=\left\{A_{i}, B_{0}, C_{i}\right\}$ for $i \neq 0 \bmod r$, then $\pi_{3} \cap \mathcal{A}_{X}=\left\{C_{i}\right\}$ is the desired singleton.

Finally, we need to show that $\pi$ is independent. Up to interchanging the roles of $B_{j}$ and $C_{j}$, we only have to check three cases: If $Y_{1}:=A_{0} \cap A_{i} \cap B_{j}$ where $i, j>0$, then we have

$$
r\left(Y_{1}\right)=r\left(A_{0} \cap \ldots \cap A_{r-1} \cap B_{j}\right)=r\left(A_{0} \cap \ldots \cap A_{r-1}\right)+1=3 .
$$

If $Y_{2}:=A_{0} \cap B_{0} \cap B_{i}$ for $i>0$, then as in the case for $Y_{1}$, we see

$$
r\left(Y_{2}\right)=r\left(A_{0} \cap B_{0} \cap \ldots \cap B_{r-1}\right)=r\left(B_{0} \cap \ldots \cap B_{r-1}\right)+1=3
$$

and likewise if $Y_{3}:=A_{0} \cap C_{0} \cap C_{i}$ for $i>0$, then we get

$$
r\left(Y_{3}\right)=r\left(A_{0} \cap C_{0} \cap \ldots \cap C_{r-1}\right)=r\left(C_{0} \cap \ldots \cap C_{r-1}\right)+1=3 .
$$

Finally, for $i>0$, it is immediate that $A_{0} \cap B_{0} \cap C_{i}=\{0\}$ has rank 3 .

Jambu and Paris [JP95] already observed that the reflection arrangement $\mathcal{A}(G(3,3,3))$ is factored but not inductively factored, see Remark 2.28 above. Note that $\mathcal{A}(G(3,3,3))$ is not inductively free, cf. Theorem 1.1 and Proposition 2.26. See also [HR16, Ex. 3.20].

Lemma 3.2. Let $W=G(r, r, \ell)$ for $r \geqslant 2$ and $\ell \geqslant 4$. Then $\mathcal{A}(W)$ is not nice. In particular, the Coxeter arrangement of $W\left(D_{\ell}\right)=G(2,2, \ell)$ for $\ell \geqslant 4$ is not nice.

Proof. Let $\ell \geqslant 4$ and $r \geqslant 2$. It suffices to show the result for $G(r, r, 4)$. For, let $W=$ $G(r, r, \ell)$ for $\ell \geqslant 5$. Then noting that $G(r, r, 4)$ is a parabolic subgroup of $W$, the result follows for $W$ from Lemma 2.34.

So let $r \geqslant 2, W=G(r, r, 4)$ and $\mathcal{A}=\mathcal{A}(W)$. We denote the coordinate functions of $\mathbb{C}^{4}$ by $x, y, z$ and $t$. Furthermore, let $\zeta$ be a primitive $r$-th root of 1 . Out of six families of hyperplanes in $\mathcal{A}$ we consider the following four, given as follows. For $i=0, \ldots, r-1$, set

$$
A_{i}:=\operatorname{ker}\left(x-\zeta^{i} y\right), B_{i}:=\operatorname{ker}\left(z-\zeta^{i} t\right), C_{i}=\operatorname{ker}\left(x-\zeta^{i} z\right) \text {, and } \quad D_{i}:=\operatorname{ker}\left(y-\zeta^{i} t\right) .
$$


We also consider the hyperplanes $A_{i}, B_{i}, C_{i}$ and $D_{i}$ for $i \in \mathbb{Z}$ simply by taking $i$ modulo $r$.

One readily checks that the following subarrangements $\mathcal{A}_{X}$ of $\mathcal{A}$ define members $X$ of rank 2 of $L(\mathcal{A})$ :

$$
\begin{gathered}
\left\{A_{0}, A_{1}, \ldots, A_{r-1}\right\},\left\{B_{0}, B_{1}, \ldots, B_{r-1}\right\},\left\{C_{0}, C_{1}, \ldots, C_{r-1}\right\},\left\{D_{0}, D_{1}, \ldots, D_{r-1}\right\}, \\
\left\{A_{i}, B_{j} \mid i, j \in\{0, \ldots, r-1\}\right\} \text { and },\left\{C_{i}, D_{j} \mid i, j \in\{0, \ldots, r-1\}\right\} .
\end{gathered}
$$

Suppose $\pi=\left(\pi_{1}, \pi_{2}, \pi_{3}, \pi_{4}\right)$ is a factorization of $\mathcal{A}$. The singleton condition from Definition 2.12(ii) applied to $X=A_{i} \cap B_{j}$ for $i, j \in\{0, \ldots, r-1\}$ shows that the $A_{i}$ and $B_{j}$ are in distinct parts of $\pi$. Likewise, the singleton condition applied to $X=$ $A_{0} \cap A_{1} \cap \ldots \cap A_{r-1}$ shows that not all the $A_{i}$ are in the same part of $\pi$. The same holds for $B_{0} \cap B_{1} \cap \ldots \cap B_{r-1}$. Thus, since $r \geqslant 2$, each part $\pi_{i}$ contains at least one of the hyperplanes $A_{0}, A_{1}, \ldots, A_{r-1}, B_{0}, B_{1}, \ldots, B_{r-1}$.

Applying the same argument to the hyperplanes $C_{0}, C_{1}, \ldots, C_{r-1}, D_{0}, D_{1}, \ldots, D_{r-1}$ implies that each part of $\pi$ contains at least two hyperplanes. This contradicts the fact that $\pi$ admits a part of cardinality 1 , cf. Remark 2.13(iii). Thus $\mathcal{A}$ does not admit a nice partition.

In [Ter92], Terao already pointed out that the Coxeter arrangement of $W\left(D_{4}\right)=$ $G(2,2,4)$ does not admit a factorization, see Remark 2.27 above.

Lemma 3.3. Let $W$ be of type $G_{33}, G_{34}, E_{6}, E_{7}$, or $E_{8}$. Then $\mathcal{A}(W)$ is not nice.

Proof. Let $W$ be as in the statement. Then $W$ admits a parabolic subgroup of type $D_{4}$; for $G_{33}, G_{34}$, see [OT92, Table C.14 - C.23]. By Lemma 3.2, $\mathcal{A}\left(W\left(D_{4}\right)\right)$ is not nice. It thus follows from Lemma 2.34 that $\mathcal{A}(W)$ is not nice either.

Lemma 3.4. Let $W$ be one of $H_{3}, G_{25}, H_{4}$, or $G_{32}$. Then $\mathcal{A}(W)$ is not nice.

Proof. First we show that $\mathcal{A}\left(H_{3}\right)$ and $\mathcal{A}\left(G_{25}\right)$ are not nice. It then follows from Lemma 2.34 and [OT92, Table C.13] that $\mathcal{A}\left(H_{4}\right)$ and $\mathcal{A}\left(G_{32}\right)$ aren't nice either.

First let $\mathcal{A}=\mathcal{A}\left(H_{3}\right)$ and let $x, y$ and $z$ be the variables in $S$ and let $\zeta$ be a primitive 5 th root of unity. We have

$$
\begin{aligned}
Q\left(H_{3}\right) & =x y z(x+y)(y+z)\left(x-\left(\zeta^{3}+\zeta^{2}\right) y\right)\left(x-\left(\zeta^{3}+\zeta^{2}+1\right) y\right) \\
& \left(x-\left(\zeta^{3}+\zeta^{2}\right) y-\left(\zeta^{3}+\zeta^{2}\right) z\right)\left(x-\left(\zeta^{3}+\zeta^{2}+1\right) y-\left(\zeta^{3}+\zeta^{2}+1\right) z\right) \\
& (x+y+z)\left(x-\left(\zeta^{3}+\zeta^{2}\right) y-\left(\zeta^{3}+\zeta^{2}+1\right) z\right)\left(x-\left(\zeta^{3}+\zeta^{2}\right) y+z\right) \\
& \left(x+y+\left(\zeta^{3}+\zeta^{2}+2\right) z\right)\left(x+y-\left(\zeta^{3}+\zeta^{2}+1\right) z\right) \\
& \left(x-2\left(\zeta^{3}+\zeta^{2}+1\right) y-\left(\zeta^{3}+\zeta^{2}+1\right) z\right) .
\end{aligned}
$$

Set

$$
\begin{aligned}
& H_{1}=\operatorname{ker} x, H_{2}=\operatorname{ker} y, H_{3}=\operatorname{ker} z, H_{4}=\operatorname{ker}\left(x-\left(\zeta^{3}+\zeta^{2}\right) y-\left(\zeta^{3}+\zeta^{2}\right) z\right), \\
& H_{5}=\operatorname{ker}\left(x+y+\left(\zeta^{3}+\zeta^{2}+2\right) z\right), \text { and } H_{6}=\operatorname{ker}\left(x-2\left(\zeta^{3}+\zeta^{2}+1\right) y-\left(\zeta^{3}+\zeta^{2}+1\right) z\right) .
\end{aligned}
$$


One checks that each of the following intersections describes a rank 2 member $X \in L(\mathcal{A})$ with $\left|\mathcal{A}_{X}\right|=2$ : $H_{1} \cap H_{3}, H_{1} \cap H_{6}, H_{3} \cap H_{6}, H_{2} \cap H_{4}, H_{2} \cap H_{5}$, and $H_{4} \cap H_{5}$. Suppose $\pi$ is a factorization of $\mathcal{A}$. Then applying the singleton condition of Definition 2.12(ii) to each of these elements of $L(\mathcal{A})$ implies that each of $H_{1}, H_{3}, H_{6}$, respectively each of $H_{2}, H_{4}, H_{5}$ must belong to a distinct part of $\pi$. However, as one of the parts of $\pi$ has to have cardinality 1 , cf. Remark 2.13(iii), this is a contradiction. Consequently, $\mathcal{A}$ is not nice.

Next let $\mathcal{A}=\mathcal{A}\left(G_{25}\right)$ and again let $x, y$ and $z$ be the variables in $S$ and let $\zeta$ be a primitive 3 rd root of unity. We have

$$
\begin{aligned}
Q\left(G_{25}\right) & =x y z(x+y+z)(x+y+\zeta z)(x+y-(\zeta+1) z) \\
& (x+\zeta y+z)(x+\zeta y+\zeta z)(x+\zeta y-(\zeta+1) z) \\
& (x-(\zeta+1) y+z)(x-(\zeta+1) y+\zeta z)(x-(\zeta+1) y-(\zeta+1) z) .
\end{aligned}
$$

Set $H_{1}=\operatorname{ker}(x+y+z), H_{2}=\operatorname{ker}(x+y+\zeta z), H_{3}=\operatorname{ker}(x+\zeta y+z), H_{4}=\operatorname{ker}(x+$ $\zeta y-(\zeta+1) z), H_{5}=\operatorname{ker}(x-(\zeta+1) y+\zeta z)$, and $H_{6}=\operatorname{ker}(x-(\zeta+1) y-(\zeta+1) z)$. One checks that each of the following intersections describes a rank 2 member $X \in L(\mathcal{A})$ with $\left|\mathcal{A}_{X}\right|=2: H_{1} \cap H_{4}, H_{1} \cap H_{5}, H_{4} \cap H_{5}, H_{2} \cap H_{3}, H_{2} \cap H_{6}$, and $H_{3} \cap H_{6}$. Suppose $\pi$ is a factorization of $\mathcal{A}$. Then, as above, applying the singleton condition of Definition 2.12(ii) to each of these elements of $L(\mathcal{A})$ implies that each of $H_{1}, H_{4}, H_{5}$, respectively each of $H_{2}, H_{3}, H_{6}$ must belong to a distinct part of $\pi$. However, as one of the parts of $\pi$ has to have cardinality 1 , cf. Remark 2.13(iii), this is a contradiction. Consequently, $\mathcal{A}$ is not nice.

Lemma 3.5. Let $W=G_{24}$. Then $\mathcal{A}(W)$ is not nice.

Proof. Let $\mathcal{A}=\mathcal{A}\left(G_{24}\right)$ and let $x, y$ and $z$ be the variables in $S$ and let $\zeta$ be a primitive 7 th root of unity. We have

$$
\begin{aligned}
& Q\left(G_{24}\right)=\left(x+\left(2 \zeta^{4}+2 \zeta^{2}+2 \zeta+1\right) y\right)\left(x+\left(-2 \zeta^{4}-2 \zeta^{2}-2 \zeta-1\right) y\right) \\
&\left(3 x+\left(-\zeta^{4}-\zeta^{2}-\zeta+3\right) y-2\left(\zeta^{4}+\zeta^{2}+\zeta\right) z\right) x \\
&\left(3 x-\left(\zeta^{4}+\zeta^{2}+\zeta+4\right) y-2\left(\zeta^{4}+\zeta^{2}+\zeta+1\right) z\right) \\
&(3 x-7 y+4 z)(3 x+7 y-4 z) \\
&\left(3 x+\left(2 \zeta^{4}+2 \zeta^{2}+2 \zeta+1\right) y-2\left(\zeta^{4}+\zeta^{2}+\zeta-1\right) z\right) \\
&\left(7 y+\left(-3 \zeta^{4}-3 \zeta^{2}-3 \zeta+2\right) z\right) \\
&\left(3 x+\left(-2 \zeta^{4}-2 \zeta^{2}-2 \zeta-1\right) y-4\left(\zeta^{4}+\zeta^{2}+\zeta-1\right) z\right) \\
&\left(3 x+\left(\zeta^{4}+\zeta^{2}+\zeta+4\right) y+2\left(\zeta^{4}+\zeta^{2}+\zeta+1\right) z\right) \\
&\left(3 x+\left(\zeta^{4}+\zeta^{2}+\zeta-3\right) y+2\left(\zeta^{4}+\zeta^{2}+\zeta\right) z\right) \\
&\left(7 y+\left(3 \zeta^{4}+3 \zeta^{2}+3 \zeta+5\right) z\right) \\
&\left(3 x+\left(2 \zeta^{4}+2 \zeta^{2}+2 \zeta+1\right) y-2\left(\zeta^{4}+\zeta^{2}+\zeta+2\right) z\right) \\
&\left(3 x+\left(-2 \zeta^{4}-2 \zeta^{2}-2 \zeta-1\right) y+2\left(\zeta^{4}+\zeta^{2}+\zeta+2\right) z\right) \\
&\left(7 y+\left(-6 \zeta^{4}-6 \zeta^{2}-6 \zeta-10\right) z\right)
\end{aligned}
$$




$$
\begin{aligned}
& \left(3 x+\left(-2 \zeta^{4}-2 \zeta^{2}-2 \zeta-1\right) y+2\left(\zeta^{4}+\zeta^{2}+\zeta-1\right) z\right) \\
& \left(3 x+\left(-2 \zeta^{4}-2 \zeta^{2}-2 \zeta-1\right) y-4\left(\zeta^{4}+\zeta^{2}+\zeta+2\right) z\right) \\
& \left(3 x+\left(2 \zeta^{4}+2 \zeta^{2}+2 \zeta+1\right) y+4\left(\zeta^{4}+\zeta^{2}+\zeta+2\right) z\right) \\
& \left(3 x+\left(2 \zeta^{4}+2 \zeta^{2}+2 \zeta+1\right) y+4\left(\zeta^{4}+\zeta^{2}+\zeta-1\right) z\right) \\
& \left(7 y+\left(6 \zeta^{4}+6 \zeta^{2}+6 \zeta-4\right) z\right) .
\end{aligned}
$$

For simplicity, we enumerate the members of $\mathcal{A}$ in the order they appear as factors in $Q\left(G_{24}\right)$, i.e., $H_{1}=\operatorname{ker}\left(x+\left(2 \zeta^{4}+2 \zeta^{2}+2 \zeta+1\right) y\right), H_{2}=\operatorname{ker}\left(x+\left(-2 \zeta^{4}-2 \zeta^{2}-2 \zeta-1\right) y\right)$, etc. Suppose $\mathcal{A}$ admits a nice partition $\pi=\left\{\pi_{1}, \pi_{2}, \pi_{3}\right\}$. Since $W$ acts transitively on $\mathcal{A}$, we may assume that $\pi_{1}=\left\{H_{1}\right\}$. The following four subsets $\mathcal{A}_{X}$ of $\mathcal{A}$ describe rank 2 members $X$ of $L(\mathcal{A}):\left\{H_{1}, H_{6}, H_{11}, H_{15}\right\},\left\{H_{1}, H_{7}, H_{12}, H_{17}\right\},\left\{H_{1}, H_{8}, H_{10}, H_{13}\right\}$, $\left\{H_{1}, H_{9}, H_{14}, H_{18}\right\},\left\{H_{1}, H_{2}, H_{4}\right\},\left\{H_{1}, H_{3}, H_{5}\right\},\left\{H_{1}, H_{16}, H_{20}\right\}$ and $\left\{H_{1}, H_{19}, H_{21}\right\}$. It follows from Corollary 2.15(iii) that each of $A:=\left\{H_{6}, H_{11}, H_{15}\right\}, B:=\left\{H_{7}, H_{12}, H_{17}\right\}, C:=$ $\left\{H_{8}, H_{10}, H_{13}\right\}, D:=\left\{H_{9}, H_{14}, H_{18}\right\}, E:=\left\{H_{2}, H_{4}\right\}, F:=\left\{H_{3}, H_{5}\right\}, G:=\left\{H_{16}, H_{20}\right\}$ and $H:=\left\{H_{19}, H_{21}\right\}$ has to be contained in one part of $\pi$.

Clearly, as only $\pi_{2}$ and $\pi_{3}$ are candidates, at least two of the sets $A, B, C, D$ have to be in one part. Applying the singleton condition in Definition 2.12(ii) to each of $\mathcal{A}_{X_{1}}=$ $\left\{H_{6}, H_{9}, H_{10}\right\}, \mathcal{A}_{X_{2}}=\left\{H_{6}, H_{14}, H_{17}\right\}, \mathcal{A}_{X_{3}}=\left\{H_{7}, H_{8}, H_{15}\right\}$ and $\mathcal{A}_{X_{4}}=\left\{H_{7}, H_{13}, H_{18}\right\}$, we conclude that

$$
\begin{aligned}
& A \cup B \in \pi_{2} \quad \Leftrightarrow \quad C \cup D \in \pi_{3}, \\
& A \cup C \in \pi_{2} \quad \Leftrightarrow \quad B \cup D \in \pi_{3}, \text { and } \\
& A \cup D \in \pi_{2} \quad \Leftrightarrow \quad B \cup C \in \pi_{3} .
\end{aligned}
$$

As the cardinality of the union of two sets in $\{A, B, C, D\}$ is even and as each of the remaining pairs of hyperplanes $\{E, F, G, H\}$ has to be added to either $\pi_{2}$ or $\pi_{3}$, it follows that $\left|\pi_{2}\right|$ is even. But this is a contradiction, since $\left|\pi_{2}\right| \in\{9,11\}$ is odd (as $\exp \mathcal{A}\left(G_{24}\right)=$ $\{1,9,11\}$, cf. Remark 2.17). It follows that $\mathcal{A}(W)$ is not nice, as claimed.

Lemma 3.6. Let $W=G_{26}$. Then $\mathcal{A}(W)$ is not nice.

Proof. Let $\mathcal{A}=\mathcal{A}\left(G_{26}\right)$ and let $x, y$ and $z$ be the variables in $S$ and let $\zeta$ be a primitive 3rd root of unity. We have

$$
\begin{aligned}
Q\left(G_{26}\right) & =x y z(x-y)(x-\zeta y)\left(x-\zeta^{2} y\right)(x-z)(x-\zeta z)\left(x-\zeta^{2} z\right) \\
& (y-z)(y-\zeta z)\left(y-\zeta^{2} z\right)(x+y+z)(x+\zeta y+z)\left(x+\zeta^{2} y+z\right) \\
& (x+y+\zeta z)\left(x+y+\zeta^{2} z\right)\left(x+\zeta^{2} y+\zeta^{2} z\right)\left(x+\zeta y+\zeta^{2} z\right) \\
& \left(x+\zeta^{2} y+\zeta z\right)(x+\zeta y+\zeta z) .
\end{aligned}
$$

Again, for simplicity, we enumerate the members of $\mathcal{A}$ in the order they appear as factors in $Q\left(G_{26}\right)$, i.e., $H_{1}=\operatorname{ker} x, H_{2}=\operatorname{ker} y$, etc. Suppose $\mathcal{A}$ admits a nice partition $\pi=$ $\left\{\pi_{1}, \pi_{2}, \pi_{3}\right\}$. There are two $W$-orbits in $\mathcal{A}$, represented by $H_{1}$ and $H_{4}$. So we may assume that the singleton of $\pi$ is either $\pi_{1}=\left\{H_{1}\right\}$ or $\pi_{1}=\left\{H_{4}\right\}$, respectively. Without loss, we 
may assume that $H_{5} \in \pi_{2}$. Consider the rank 2 elements $X_{1}, \ldots, X_{4}$ of $L(\mathcal{A})$ given by $\mathcal{A}_{X_{1}}=\left\{H_{5}, H_{3}\right\}, \mathcal{A}_{X_{2}}=\left\{H_{5}, H_{14}\right\}, \mathcal{A}_{X_{3}}=\left\{H_{5}, H_{19}\right\}$ and $\mathcal{A}_{X_{4}}=\left\{H_{5}, H_{21}\right\}$, respectively. It follows from Corollary 2.15(iii) that $H_{3}, H_{14}, H_{19}, H_{21} \in \pi_{3}$. But this is a contradiction to Remark 2.13(iii), since $\mathcal{A}_{H_{3} \cap H_{14} \cap H_{19} \cap H_{21}}=\left\{H_{3}, H_{14}, H_{19}, H_{21}\right\}$. It follows that $\mathcal{A}(W)$ is not nice, as desired.

Lemma 3.7. Let $W=G_{27}$. Then $\mathcal{A}(W)$ is not nice.

Proof. Let $\mathcal{A}=\mathcal{A}\left(G_{27}\right)$ and let $x, y$ and $z$ be the variables in $S$ and let $\zeta$ be a primitive 15 th root of unity. We have

$$
\begin{aligned}
& Q\left(G_{27}\right)=\left(15 x+\left(24 \zeta^{7}-18 \zeta^{6}-6 \zeta^{5}+18 \zeta^{4}-6 \zeta^{3}+12 \zeta^{2}-6 \zeta-15\right) y\right. \\
& \left.+\left(-4 \zeta^{7}+8 \zeta^{6}-4 \zeta^{5}+2 \zeta^{4}-4 \zeta^{3}-12 \zeta^{2}+6 \zeta\right) z\right) \\
& \left(3 y+\left(\zeta^{7}+3 \zeta^{5}-\zeta^{4}-\zeta^{3}+\zeta^{2}-\zeta+2\right) z\right) \\
& \left(15 x+\left(-18 \zeta^{6}+6 \zeta^{5}+12 \zeta^{4}-6 \zeta^{3}+12 \zeta^{2}-24 \zeta-3\right) y\right. \\
& \left.+\left(-12 \zeta^{6}+4 \zeta^{5}+8 \zeta^{4}-4 \zeta^{3}+8 \zeta^{2}-16 \zeta-2\right) z\right) \\
& \left(15 x+\left(-12 \zeta^{7}-18 \zeta^{6}+12 \zeta^{5}-6 \zeta^{4}-6 \zeta^{3}+12 \zeta^{2}-18 \zeta+3\right) y\right. \\
& \left.+\left(2 \zeta^{7}+8 \zeta^{6}-2 \zeta^{5}+6 \zeta^{4}+6 \zeta^{3}-2 \zeta^{2}-2 \zeta+2\right) z\right) \\
& \left(15 x+\left(12 \zeta^{7}-18 \zeta^{6}-6 \zeta^{3}+12 \zeta^{2}-9\right) y\right. \\
& \left.+\left(-2 \zeta^{7}+28 \zeta^{6}-10 \zeta^{5}+6 \zeta^{3}-22 \zeta^{2}+20 \zeta+4\right) z\right) \\
& \left(15 x+\left(9 \zeta^{7}-12 \zeta^{6}-3 \zeta^{5}+9 \zeta^{4}-9 \zeta^{3}+3 \zeta^{2}-3 \zeta-9\right) y\right. \\
& \left.+\left(\zeta^{7}-8 \zeta^{6}+3 \zeta^{5}-4 \zeta^{4}-\zeta^{3}+7 \zeta^{2}-2 \zeta-1\right) z\right) \\
& \left(15 x+\left(12 \zeta^{7}-18 \zeta^{6}-6 \zeta^{3}+12 \zeta^{2}-9\right) y\right. \\
& \left.+\left(-22 \zeta^{7}+8 \zeta^{6}+10 \zeta^{5}+6 \zeta^{3}-2 \zeta^{2}-20 \zeta+14\right) z\right) \\
& \left(15 x+\left(6 \zeta^{7}+6 \zeta^{6}+12 \zeta^{3}+6 \zeta^{2}+3\right) y\right. \\
& \left.+\left(14 \zeta^{7}-16 \zeta^{6}+10 \zeta^{4}-2 \zeta^{3}+14 \zeta^{2}-10 \zeta-8\right) z\right) \\
& \left(15 x+\left(-3 \zeta^{7}-6 \zeta^{6}+6 \zeta^{5}-3 \zeta^{4}+3 \zeta^{3}+9 \zeta^{2}-9 \zeta+3\right) y\right. \\
& \left.+\left(3 \zeta^{7}+6 \zeta^{6}-\zeta^{5}-2 \zeta^{4}+7 \zeta^{3}+\zeta^{2}+4 \zeta+2\right) z\right) \\
& \left(15 x+\left(12 \zeta^{7}+18 \zeta^{6}-12 \zeta^{5}+6 \zeta^{4}+6 \zeta^{3}-12 \zeta^{2}+18 \zeta-3\right) y\right. \\
& \left.+\left(-2 \zeta^{7}-8 \zeta^{6}+2 \zeta^{5}-6 \zeta^{4}-6 \zeta^{3}+2 \zeta^{2}+2 \zeta-2\right) z\right) \\
& \left(15 x+\left(30 \zeta^{7}-6 \zeta^{6}-18 \zeta^{5}+24 \zeta^{4}-12 \zeta^{3}-6 \zeta^{2}+12 \zeta-21\right) y\right. \\
& \left.+\left(20 \zeta^{7}-4 \zeta^{6}-12 \zeta^{5}+16 \zeta^{4}-8 \zeta^{3}-4 \zeta^{2}+8 \zeta-14\right) z\right) \\
& \left(15 x+\left(6 \zeta^{7}+6 \zeta^{6}+12 \zeta^{3}+6 \zeta^{2}+3\right) y\right. \\
& \left.+\left(-26 \zeta^{7}+4 \zeta^{6}+10 \zeta^{5}-20 \zeta^{4}-2 \zeta^{3}-6 \zeta^{2}+12\right) z\right) \\
& \left(3 y+\left(-2 \zeta^{7}-2 \zeta^{5}-\zeta^{4}+2 \zeta^{3}-2 \zeta^{2}-\zeta+1\right) z\right) \\
& \left(15 x+\left(3 \zeta^{7}+6 \zeta^{6}-6 \zeta^{5}+3 \zeta^{4}-3 \zeta^{3}-9 \zeta^{2}+9 \zeta-3\right) y\right. \\
& \left.+\left(-3 \zeta^{7}-6 \zeta^{6}+\zeta^{5}+2 \zeta^{4}-7 \zeta^{3}-\zeta^{2}-4 \zeta-2\right) z\right)
\end{aligned}
$$




$$
\begin{aligned}
& \left(x+\left(2 \zeta^{6}+2 \zeta^{3}+1\right) y\right) \\
& \left(15 x+\left(-12 \zeta^{7}+6 \zeta^{5}-3 \zeta^{4}-9 \zeta+6\right) y\right. \\
& \left.+\left(-3 \zeta^{7}-\zeta^{5}-2 \zeta^{4}-5 \zeta^{3}-5 \zeta^{2}+4 \zeta-1\right) z\right) \\
& \left(15 x+\left(-6 \zeta^{7}+6 \zeta^{6}+6 \zeta^{5}-18 \zeta^{4}+12 \zeta^{3}+6 \zeta^{2}+6 \zeta+9\right) y\right. \\
& \left.+\left(-14 \zeta^{7}+4 \zeta^{6}+4 \zeta^{5}-2 \zeta^{4}-2 \zeta^{3}-6 \zeta^{2}-6 \zeta+6\right) z\right) \\
& \left(15 x+\left(-12 \zeta^{7}+18 \zeta^{6}+6 \zeta^{3}-12 \zeta^{2}+9\right) y\right. \\
& \left.+\left(22 \zeta^{7}-8 \zeta^{6}-10 \zeta^{5}-6 \zeta^{3}+2 \zeta^{2}+20 \zeta-14\right) z\right) \\
& \left(15 x+\left(-6 \zeta^{7}-6 \zeta^{6}-12 \zeta^{3}-6 \zeta^{2}-3\right) y\right. \\
& \left.+\left(-14 \zeta^{7}+16 \zeta^{6}-10 \zeta^{4}+2 \zeta^{3}-14 \zeta^{2}+10 \zeta+8\right) z\right) \\
& \left(x+\left(2 \zeta^{7}-2 \zeta^{6}+2 \zeta^{2}-1\right) y\right)\left(x+\left(-2 \zeta^{6}-2 \zeta^{3}-1\right) y\right) \\
& \left(3 y+\left(-\zeta^{7}+2 \zeta^{5}+\zeta^{4}+\zeta^{3}-\zeta^{2}+\zeta+2\right) z\right) \\
& \left(15 x+\left(6 \zeta^{7}+6 \zeta^{6}+12 \zeta^{3}+6 \zeta^{2}+3\right) y\right. \\
& \left.+\left(14 \zeta^{7}-16 \zeta^{6}-10 \zeta^{5}+20 \zeta^{4}-22 \zeta^{3}-6 \zeta^{2}-18\right) z\right) \\
& \left(15 x+\left(-6 \zeta^{7}+3 \zeta^{5}-9 \zeta^{4}+3 \zeta+3\right) y\right. \\
& \left.+\left(11 \zeta^{7}-10 \zeta^{6}-3 \zeta^{5}+4 \zeta^{4}-5 \zeta^{3}+5 \zeta^{2}+2 \zeta-8\right) z\right) \\
& \left(15 x+\left(18 \zeta^{6}-6 \zeta^{5}-12 \zeta^{4}+6 \zeta^{3}-12 \zeta^{2}+24 \zeta+3\right) y\right. \\
& \left.+\left(12 \zeta^{6}-4 \zeta^{5}-8 \zeta^{4}+4 \zeta^{3}-8 \zeta^{2}+16 \zeta+2\right) z\right) \\
& \left(15 x+\left(-6 \zeta^{7}-6 \zeta^{6}-12 \zeta^{3}-6 \zeta^{2}-3\right) y\right. \\
& \left.+\left(26 \zeta^{7}-4 \zeta^{6}-10 \zeta^{5}+20 \zeta^{4}+2 \zeta^{3}+6 \zeta^{2}-12\right) z\right) \\
& \left(15 x+\left(-12 \zeta^{7}+18 \zeta^{6}+6 \zeta^{3}-12 \zeta^{2}+9\right) y\right. \\
& \left.+\left(-18 \zeta^{7}+12 \zeta^{6}+10 \zeta^{5}-10 \zeta^{4}+14 \zeta^{3}+2 \zeta^{2}-10 \zeta+16\right) z\right) \\
& \left(15 x+\left(-9 \zeta^{7}+12 \zeta^{6}+3 \zeta^{5}-9 \zeta^{4}+9 \zeta^{3}-3 \zeta^{2}+3 \zeta+9\right) y\right. \\
& \left.+\left(-\zeta^{7}+8 \zeta^{6}-3 \zeta^{5}+4 \zeta^{4}+\zeta^{3}-7 \zeta^{2}+2 \zeta+1\right) z\right) \\
& x\left(x+\left(-2 \zeta^{7}+2 \zeta^{6}-2 \zeta^{2}+1\right) y\right) \\
& \left(15 x+\left(-24 \zeta^{7}+18 \zeta^{6}+6 \zeta^{5}-18 \zeta^{4}+6 \zeta^{3}-12 \zeta^{2}+6 \zeta+15\right) y\right. \\
& \left.+\left(4 \zeta^{7}-8 \zeta^{6}+4 \zeta^{5}-2 \zeta^{4}+4 \zeta^{3}+12 \zeta^{2}-6 \zeta\right) z\right) \\
& \left(15 x+\left(-18 \zeta^{7}+6 \zeta^{6}+12 \zeta^{5}-6 \zeta^{4}+12 \zeta^{3}+6 \zeta^{2}-18 \zeta+15\right) y\right. \\
& \left.+\left(8 \zeta^{7}-16 \zeta^{6}-2 \zeta^{5}+6 \zeta^{4}-12 \zeta^{3}+4 \zeta^{2}-2 \zeta-10\right) z\right) \\
& \left(15 x+\left(6 \zeta^{7}-3 \zeta^{5}+9 \zeta^{4}-3 \zeta-3\right) y\right. \\
& \left.+\left(-11 \zeta^{7}+10 \zeta^{6}+3 \zeta^{5}-4 \zeta^{4}+5 \zeta^{3}-5 \zeta^{2}-2 \zeta+8\right) z\right) \\
& \left(15 x+\left(18 \zeta^{7}-6 \zeta^{6}-12 \zeta^{5}+6 \zeta^{4}-12 \zeta^{3}-6 \zeta^{2}+18 \zeta-15\right) y\right. \\
& \left.+\left(-8 \zeta^{7}+16 \zeta^{6}+2 \zeta^{5}-6 \zeta^{4}+12 \zeta^{3}-4 \zeta^{2}+2 \zeta+10\right) z\right) \\
& \left(15 x+\left(-6 \zeta^{7}-6 \zeta^{6}-12 \zeta^{3}-6 \zeta^{2}-3\right) y\right. \\
& \left.+\left(-4 \zeta^{7}-4 \zeta^{6}+10 \zeta^{4}-8 \zeta^{3}-4 \zeta^{2}-10 \zeta-2\right) z\right)
\end{aligned}
$$




$$
\begin{aligned}
(15 x & +\left(-12 \zeta^{7}+18 \zeta^{6}+6 \zeta^{3}-12 \zeta^{2}+9\right) y \\
& \left.+\left(12 \zeta^{7}+12 \zeta^{6}-10 \zeta^{5}+10 \zeta^{4}+4 \zeta^{3}-8 \zeta^{2}+10 \zeta-4\right) z\right) \\
(15 x & +\left(-12 \zeta^{7}+18 \zeta^{6}+6 \zeta^{3}-12 \zeta^{2}+9\right) y \\
& \left.+\left(2 \zeta^{7}-28 \zeta^{6}+10 \zeta^{5}-6 \zeta^{3}+22 \zeta^{2}-20 \zeta-4\right) z\right) \\
(15 x & +\left(-30 \zeta^{7}+6 \zeta^{6}+18 \zeta^{5}-24 \zeta^{4}+12 \zeta^{3}+6 \zeta^{2}-12 \zeta+21\right) y \\
& \left.+\left(-20 \zeta^{7}+4 \zeta^{6}+12 \zeta^{5}-16 \zeta^{4}+8 \zeta^{3}+4 \zeta^{2}-8 \zeta+14\right) z\right) \\
(15 x & +\left(6 \zeta^{7}+6 \zeta^{6}+12 \zeta^{3}+6 \zeta^{2}+3\right) y \\
& \left.+\left(4 \zeta^{7}+4 \zeta^{6}-10 \zeta^{4}+8 \zeta^{3}+4 \zeta^{2}+10 \zeta+2\right) z\right) \\
(15 x & +\left(12 \zeta^{7}-18 \zeta^{6}-6 \zeta^{3}+12 \zeta^{2}-9\right) y \\
& \left.+\left(-12 \zeta^{7}-12 \zeta^{6}+10 \zeta^{5}-10 \zeta^{4}-4 \zeta^{3}+8 \zeta^{2}-10 \zeta+4\right) z\right) \\
(15 x+ & \left(-6 \zeta^{7}-6 \zeta^{6}-12 \zeta^{3}-6 \zeta^{2}-3\right) y \\
& \left.+\left(-14 \zeta^{7}+16 \zeta^{6}+10 \zeta^{5}-20 \zeta^{4}+22 \zeta^{3}+6 \zeta^{2}+18\right) z\right) \\
(15 x+ & \left(12 \zeta^{7}-6 \zeta^{5}+3 \zeta^{4}+9 \zeta-6\right) y \\
& \left.+\left(3 \zeta^{7}+\zeta^{5}+2 \zeta^{4}+5 \zeta^{3}+5 \zeta^{2}-4 \zeta+1\right) z\right) \\
(15 x+ & \left(6 \zeta^{7}-6 \zeta^{6}-6 \zeta^{5}+18 \zeta^{4}-12 \zeta^{3}-6 \zeta^{2}-6 \zeta-9\right) y \\
& \left.+\left(14 \zeta^{7}-4 \zeta^{6}-4 \zeta^{5}+2 \zeta^{4}+2 \zeta^{3}+6 \zeta^{2}+6 \zeta-6\right) z\right) \\
(15 x+ & \left(12 \zeta^{7}-18 \zeta^{6}-6 \zeta^{3}+12 \zeta^{2}-9\right) y \\
& \left.+\left(18 \zeta^{7}-12 \zeta^{6}-10 \zeta^{5}+10 \zeta^{4}-14 \zeta^{3}-2 \zeta^{2}+10 \zeta-16\right) z\right) \\
(3 y+ & \left.\left(2 \zeta^{7}-3 \zeta^{5}+\zeta^{4}-2 \zeta^{3}+2 \zeta^{2}+\zeta-2\right) z\right) .
\end{aligned}
$$

For simplicity, once again we enumerate the members of $\mathcal{A}$ in the order they appear as factors in $Q\left(G_{27}\right)$. Suppose $\mathcal{A}$ admits a nice partition $\pi=\left\{\pi_{1}, \pi_{2}, \pi_{3}\right\}$. Since $W$ acts transitively on $\mathcal{A}(W)$, we can assume without loss that $\pi_{1}=\left\{H_{1}\right\}$. We derive a contradiction by considering the following 25 rank 2 members $X_{i}$ of $L(\mathcal{A})$ :

$$
\begin{aligned}
& \mathcal{A}_{X_{1}}=\left\{H_{1}, H_{2}, H_{3}, H_{4}, H_{5}\right\}, \quad \mathcal{A}_{X_{2}}=\left\{H_{1}, H_{6}, H_{7}, H_{8}, H_{9}\right\}, \\
& \mathcal{A}_{X_{3}}=\left\{H_{1}, H_{10}, H_{11}, H_{12}, H_{13}\right\}, \quad \mathcal{A}_{X_{4}}=\left\{H_{1}, H_{14}, H_{15}, H_{16}, H_{17}\right\} \text {, } \\
& \mathcal{A}_{X_{5}}=\left\{H_{4}, H_{6}, H_{18}\right\}, \quad \mathcal{A}_{X_{6}}=\left\{H_{3}, H_{9}, H_{19}\right\}, \\
& \mathcal{A}_{X_{7}}=\left\{H_{2}, H_{7}, H_{20}\right\}, \quad \mathcal{A}_{X_{8}}=\left\{H_{5}, H_{8}, H_{21}\right\}, \\
& \mathcal{A}_{X_{9}}=\left\{H_{3}, H_{7}, H_{22}\right\}, \quad \mathcal{A}_{X_{10}}=\left\{H_{5}, H_{6}, H_{23}\right\}, \\
& \mathcal{A}_{X_{11}}=\left\{H_{19}, H_{23}, H_{24}\right\}, \quad \mathcal{A}_{X_{12}}=\left\{H_{18}, H_{22}, H_{25}\right\} \text {, } \\
& \mathcal{A}_{X_{13}}=\left\{H_{21}, H_{22}, H_{26}\right\}, \quad \mathcal{A}_{X_{14}}=\left\{H_{20}, H_{23}, H_{27}\right\}, \\
& \mathcal{A}_{X_{15}}=\left\{H_{24}, H_{25}, H_{26}, H_{27}\right\}, \quad \mathcal{A}_{X_{16}}=\left\{H_{11}, H_{17}, H_{18}\right\}, \\
& \mathcal{A}_{X_{17}}=\left\{H_{11}, H_{16}, H_{20}\right\}, \quad \mathcal{A}_{X_{18}}=\left\{H_{10}, H_{16}, H_{21}\right\}, \\
& \mathcal{A}_{X_{19}}=\left\{H_{10}, H_{17}, H_{19}\right\}, \quad \mathcal{A}_{X_{20}}=\left\{H_{12}, H_{15}, H_{22}\right\}, \\
& \mathcal{A}_{X_{21}}=\left\{H_{13}, H_{15}, H_{23}\right\}, \quad \mathcal{A}_{X_{22}}=\left\{H_{4}, H_{12}, H_{25}\right\}, \\
& \mathcal{A}_{X_{23}}=\left\{H_{8}, H_{14}, H_{25}\right\}, \quad \mathcal{A}_{X_{24}}=\left\{H_{3}, H_{15}, H_{24}\right\}, \\
& \mathcal{A}_{X_{25}}=\left\{H_{9}, H_{13}, H_{24}\right\} \text {. }
\end{aligned}
$$


Applying the singleton condition from Definition 2.12(ii) to $\mathcal{A}_{X_{1}}, \mathcal{A}_{X_{2}}, \mathcal{A}_{X_{3}}$, and $\mathcal{A}_{X_{4}}$ shows that each of the sets

$$
\begin{array}{ll}
A:=\left\{H_{2}, H_{3}, H_{4}, H_{5}\right\}, & B:=\left\{H_{6}, H_{7}, H_{8}, H_{9}\right\} \\
C:=\left\{H_{10}, H_{11}, H_{12}, H_{13}\right\} \text { and } & D:=\left\{H_{14}, H_{15}, H_{16}, H_{17}\right\}
\end{array}
$$

is a subset of $\pi_{2}$ or $\pi_{3}$. First suppose that $A \cup B \subset \pi_{2}$. Then the singleton condition Definition 2.12(ii) applied to each of $\mathcal{A}_{X_{5}}, \mathcal{A}_{X_{6}}, \mathcal{A}_{X_{7}}, \mathcal{A}_{X_{8}}, \mathcal{A}_{X_{9}}$, and $\mathcal{A}_{X_{10}}$ shows that $H_{18}, H_{19}, H_{20}, H_{21}, H_{22}, H_{23} \in \pi_{3}$. The same argument applied to $\mathcal{A}_{X_{11}}, \mathcal{A}_{X_{12}}, \mathcal{A}_{X_{13}}$, and $\mathcal{A}_{X_{14}}$ shows that $H_{24}, H_{25}, H_{26}, H_{27} \in \pi_{2}$. This now leads to a contradiction, since $\mathcal{A}_{X_{15}}$ then violates the singleton condition. Now suppose that $C \cup D \subset \pi_{2}$. Then Remark 2.13(iii) applied to $\mathcal{A}_{X_{16}}, \mathcal{A}_{X_{17}}, \mathcal{A}_{X_{18}}, \mathcal{A}_{X_{19}}, \mathcal{A}_{X_{20}}$, and $\mathcal{A}_{X_{21}}$ shows that $H_{18}, H_{19}, H_{20}, H_{21}$, $H_{22}, H_{23} \in \pi_{3}$. This however leads again to the same contradiction as in the case $A \cup B \subset \pi_{2}$. It follows that exactly two of the sets $A, B, C, D$ have to be contained in $\pi_{2}$. Now suppose that $A \cup C \subset \pi_{2}$ and $B \cup D \subset \pi_{3}$. The singleton condition applied to $\mathcal{A}_{X_{22}}$ and $\mathcal{A}_{X_{23}}$ implies that $H_{25} \in \pi_{2} \cap \pi_{3}$, which is absurd. Finally, suppose that $A \cup D \subset \pi_{2}$ and $B \cup C \subset \pi_{3}$. The singleton condition applied to $\mathcal{A}_{X_{24}}$ and $\mathcal{A}_{X_{25}}$ implies that $H_{24} \in \pi_{2} \cap \pi_{3}$, which is absurd. It follows that $\mathcal{A}\left(G_{27}\right)$ is not nice.

Lemma 3.8. Let $W$ be of type $F_{4}$. Then $\mathcal{A}(W)$ is not nice.

Proof. Let $\mathcal{A}=\mathcal{A}\left(F_{4}\right)$ and let $u, x, y$ and $z$ be the variables in $S$. We have

$$
\begin{aligned}
Q\left(F_{4}\right) & =u x y z(u+x)(x+y)(y+z)(u+x+y)(x+2 y)(x+y+z) \\
& (u+x+2 y)(u+x+y+z)(x+2 y+z)(u+2 x+2 y)(u+x+2 y+z) \\
& (x+2 y+2 z)(u+2 x+2 y+z)(u+x+2 y+2 z)(u+2 x+3 y+z) \\
& (u+2 x+2 y+2 z)(u+2 x+3 y+2 z)(u+2 x+4 y+2 z) \\
& (u+3 x+4 y+2 z)(2 u+3 x+4 y+2 z) .
\end{aligned}
$$

For simplicity, we enumerate the members of $\mathcal{A}$ in the order they appear as factors in $Q\left(F_{4}\right)$, i.e., $H_{1}=\operatorname{ker} u, H_{2}=\operatorname{ker} x$, etc. Suppose that $\pi=\left(\pi_{1}, \pi_{2}, \pi_{3}, \pi_{4}\right)$ is a nice partition of $\mathcal{A}$. The following argument holds for every choice of a singleton $\pi_{1}=\left\{H_{i}\right\}$. Without loss, let $\pi_{1}=\left\{H_{1}\right\}$. Consider the following rank 2 members of $L(\mathcal{A})$ :

$$
\begin{aligned}
& \mathcal{A}_{X_{1}}=\left\{H_{1}, H_{2}, H_{5}\right\}, \mathcal{A}_{X_{2}}=\left\{H_{1}, H_{6}, H_{8}, H_{14}\right\}, \\
& \mathcal{A}_{X_{3}}=\left\{H_{1}, H_{10}, H_{12}, H_{20}\right\}, \text { and } \mathcal{A}_{X_{4}}=\left\{H_{1}, H_{13}, H_{15}, H_{22}\right\} .
\end{aligned}
$$

The singleton condition from Definition 2.12(ii) applied to each of $X_{1}, X_{2}, X_{3}$, and $X_{4}$ shows that each of the sets $A:=\left\{H_{2}, H_{5}\right\}, B:=\left\{H_{6}, H_{8}, H_{14}\right\}, C:=\left\{H_{10}, H_{12}, H_{20}\right\}$, and $D:=\left\{H_{13}, H_{15}, H_{22}\right\}$ is a subset of $\pi_{2}, \pi_{3}$ or $\pi_{4}$. Now, applying the singleton condition to

$$
\begin{aligned}
& \mathcal{A}_{X_{5}}=\left\{H_{2}, H_{8}\right\}, \mathcal{A}_{X_{6}}=\left\{H_{2}, H_{12}\right\}, \mathcal{A}_{X_{7}}=\left\{H_{2}, H_{13}\right\}, \\
& \mathcal{A}_{X_{8}}=\left\{H_{6}, H_{20}\right\}, \mathcal{A}_{X_{9}}=\left\{H_{6}, H_{22}\right\}, \text { and } \mathcal{A}_{X_{10}}=\left\{H_{10}, H_{22}\right\}
\end{aligned}
$$

shows that $A, B, C$ and $D$ have to be in different blocks of the factorization. This contradicts the fact that $\pi_{1}=\left\{H_{1}\right\}$. It follows that $\mathcal{A}$ is not nice. 
Lemma 3.9. Let $W=G_{29}$. Then $\mathcal{A}(W)$ is not nice.

Proof. Let $\mathcal{A}=\mathcal{A}\left(G_{29}\right)$ and let $u, x, y$ and $z$ be the variables in $S$. We have

$$
\begin{aligned}
& Q\left(G_{29}\right)=z(u-x+i y+i z)(u-x)(x-y)(u-x+i y-i z)(y+z)(u+i x-y+i z) \\
& \quad(u-x-i y-i z)(u-y)(u-x-i y+i z)(u-i x+i y+z)(u+i x-y-i z) \\
& \quad(x+z)(u-i x-y-i z)(u-i x-y+i z)(u+i x-i y+z)(y-z)(u-i x+i y-z) \\
& \quad(x-z)(u+i x-i y-z) y(u+z)(u+i x+y+i z)(u+i x+i y+z)(u+i x+i y-z) \\
& \quad(u-z)(u-i x-i y+z)(u-i x+y-i z)(u-i x+y+i z) x(u+x+i y+i z) \\
& \quad(u+x-i y-i z)(u+x-i y+i z)(u+i x+y-i z)(u-i x-i y-z) \\
& \quad(u+x+i y-i z)(x+y) u(u+y)(u+x) .
\end{aligned}
$$

Again, for simplicity, we enumerate the members of $\mathcal{A}$ in the order they appear as factors in $Q\left(G_{29}\right)$, i.e., $H_{1}=\operatorname{ker} z, H_{2}=\operatorname{ker}(u-x+i y+i z)$, etc. Suppose that $\pi=\left(\pi_{1}, \pi_{2}, \pi_{3}, \pi_{4}\right)$ is a nice partition of $\mathcal{A}$. We may assume that $\pi_{1}=\left\{H_{1}\right\}$ is the singleton, since $W$ is transitive on $\mathcal{A}$. Corollary 2.15 (iii) applied to $\mathcal{A}_{H_{1} \cap H_{6}}=\left\{H_{1}, H_{6}, H_{17}, H_{21}\right\}, \mathcal{A}_{H_{1} \cap H_{22}}=$ $\left\{H_{1}, H_{22}, H_{26}, H_{38}\right\}$ and $\mathcal{A}_{H_{1} \cap H_{13}}=\left\{H_{1}, H_{13}, H_{19}, H_{30}\right\}$ shows that each of the sets $A:=$ $\left\{H_{6}, H_{17}, H_{21}\right\}, B:=\left\{H_{22}, H_{26}, H_{38}\right\}$ and $C:=\left\{H_{13}, H_{19}, H_{30}\right\}$ is contained in one of the parts of $\pi$. Moreover, these sets are in different parts of $\pi$, thanks to Remark 2.13(iii) applied to $\mathcal{A}_{H_{6} \cap H_{38}}=\left\{H_{6}, H_{38}\right\}, \mathcal{A}_{H_{6} \cap H_{30}}=\left\{H_{6}, H_{30}\right\}$ and $\mathcal{A}_{H_{13} \cap H_{38}}=\left\{H_{13}, H_{38}\right\}$. So lets assume that $A \subset \pi_{2}, B \subset \pi_{3}$ and $C \subset \pi_{4}$. Consider $\mathcal{A}_{X}=\left\{H, H^{\prime}\right\}$, where $H \in A$ and $H^{\prime}$ is one of the following hyperplanes $H_{2}, H_{3}, H_{5}, H_{8}, H_{10}, H_{13}, H_{19}, H_{22}, H_{26}, H_{30}$, $H_{31}, H_{32}, H_{33}, H_{36}, H_{38}$ and $H_{40}$. Thanks to Definition 2.12(ii), it follows that none of the $H^{\prime}$ is contained in $\pi_{2}$. Using the same argument, considering $\mathcal{A}_{X}=\left\{H, H^{\prime}\right\}$, where this time $H \in C$ and $H^{\prime}$ is one of the following hyperplanes $H_{6}, H_{7}, H_{9}, H_{12}, H_{14}, H_{15}, H_{17}$, $H_{21}, H_{22}, H_{23}, H_{26}, H_{28}, H_{29}, H_{34}, H_{38}$ and $H_{39}$, it follows again from Definition 2.12(ii) that none of the $H^{\prime}$ is contained in $\pi_{4}$.

Next we consider the following rank 3 members $Y_{i}$ of $L(\mathcal{A})$ :

$$
\begin{aligned}
& \mathcal{A}_{Y_{1}}=\left\{H_{1}, H_{2}, H_{3}, H_{5}, H_{6}, H_{8}, H_{10}, H_{17}, H_{21}\right\}, \\
& \mathcal{A}_{Y_{2}}=\left\{H_{1}, H_{6}, H_{17}, H_{21}, H_{31}, H_{32}, H_{33}, H_{36}, H_{40}\right\}, \\
& \mathcal{A}_{Y_{3}}=\left\{H_{1}, H_{7}, H_{9}, H_{12}, H_{13}, H_{14}, H_{15}, H_{19}, H_{30}\right\}, \text { and } \\
& \mathcal{A}_{Y_{4}}=\left\{H_{1}, H_{13}, H_{19}, H_{23}, H_{28}, H_{29}, H_{30}, H_{34}, H_{39}\right\} .
\end{aligned}
$$

Now using the information above, it follows from Corollary 2.15(iii) that each of the following four sets is contained in one of the parts of $\pi$.

$$
\begin{array}{ll}
Z_{1}:=\left\{H_{2}, H_{3}, H_{5}, H_{8}, H_{10}\right\}, & Z_{2}:=\left\{H_{31}, H_{32}, H_{33}, H_{36}, H_{40}\right\}, \\
Z_{3}:=\left\{H_{7}, H_{9}, H_{12}, H_{14}, H_{15}\right\}, \text { and } & Z_{4}:=\left\{H_{23}, H_{28}, H_{29}, H_{34}, H_{39}\right\} .
\end{array}
$$

The singleton condition from Definition 2.12 applied to each of $\left\{H_{2}, H_{12}\right\},\left\{H_{2}, H_{28}\right\}$, $\left\{H_{3}, H_{31}\right\},\left\{H_{7}, H_{32}\right\},\left\{H_{7}, H_{39}\right\}$ and $\left\{H_{23}, H_{36}\right\}$ shows that each of the $Y_{i}^{\prime}$ 's lies in a different part of $\pi$. But this contradicts the fact that $\pi_{1}=\left\{H_{1}\right\}$. It follows that $\mathcal{A}$ is not nice. 
Lemma 3.10. Let $W=G_{31}$. Then $\mathcal{A}(W)$ is not nice.

Proof. Let $\mathcal{A}=\mathcal{A}\left(G_{31}\right)$ and let $u, x, y$, and $z$ be the variables in $S$. We have

$$
\begin{aligned}
& Q\left(G_{31}\right)=u(u+i x)(u-x)(u+x+y+z)(x-y)(u-i x)(u+x)(u-x-y-z) x \\
&(u-x-i y-i z)(u+i y)(u+x-i y-i z)(u-y)(u-x+y+z)(u-x+i y+i z) \\
&(x+i y)(y+z)(u-i x-y-i z)(u+x+i y+i z)(u+i x-i y+z)(u-i y)(u+y) \\
& y(u-i x+y-i z)(u+x-y+z)(u+i x-y+i z)(x-i y)(x+z)(u+i x+y+i z) \\
&(u-i x+i y+z)(u+x-y-z)(u-i x+i y-z)(u-x+y-z)(u+i x-i y-z) \\
&(u+i x+i y-z)(x+y)(u-x+i y-z)(u+i x+y-i z)(u-i z)(u+i x-y-i z) \\
& \quad(u+x+i y-i z)(u+i x+i y+z)(u+z)(u-i x-i y+z)(u-x-y+z) \\
& \quad(u-i x-y+i z)(u-i x+y+i z)(u-x-i y+i z)(u-i x-i y-z)(x-i z)(x+i z) \\
& \quad(y+i z)(u+i z)(u+x-i y+i z)(y-i z)(u-z)(u+x+y-z)(x-z) z(y-z) .
\end{aligned}
$$

Again, for simplicity, we enumerate the members of $\mathcal{A}$ in the order they appear as factors in $Q\left(G_{31}\right)$, i.e., $H_{1}=\operatorname{ker} u, H_{2}=\operatorname{ker}(u+i x)$, etc. Suppose that $\pi=\left(\pi_{1}, \pi_{2}, \pi_{3}, \pi_{4}\right)$ is a nice partition of $\mathcal{A}$. Since $G_{31}$ acts transitively on $\mathcal{A}$, we may assume that $\pi_{1}=$ $\left\{H_{1}\right\}$. Then Corollary 2.15(iii) applied to $\mathcal{A}_{H_{1} \cap H_{2}}=\left\{H_{1}, H_{2}, H_{3}, H_{6}, H_{7}, H_{9}\right\}, \mathcal{A}_{H_{1} \cap H_{11}}=$ $\left\{H_{1}, H_{11}, H_{13}, H_{21}, H_{22}, H_{23}\right\}$ and $\mathcal{A}_{H_{1} \cap H_{39}}=\left\{H_{1}, H_{39}, H_{43}, H_{53}, H_{56}, H_{59}\right\}$ shows each of $A:=\left\{H_{2}, H_{3}, H_{6}, H_{7}, H_{9}\right\}, B:=\left\{H_{11}, H_{13}, H_{21}, H_{22}, H_{23}\right\}$ and $C:=\left\{H_{39}, H_{43}, H_{53}, H_{56}\right.$, $\left.H_{59}\right\}$ is a subset of one of the parts of $\pi$. Moreover, $A, B, C$ have to be in distinct parts of $\pi$, thanks to Remark 2.13(iii) applied to $\mathcal{A}_{H_{2} \cap H_{23}}=\left\{H_{2}, H_{23}\right\}, \mathcal{A}_{H_{2} \cap H_{59}}=\left\{H_{2}, H_{59}\right\}$ and $\mathcal{A}_{H_{11} \cap H_{59}}=\left\{H_{11}, H_{59}\right\}$. But now applying the singleton condition from Remark 2.13(iii) to $\mathcal{A}_{H_{3} \cap H_{4}}=\left\{H_{3}, H_{4}\right\}, \mathcal{A}_{H_{4} \cap H_{13}}=\left\{H_{4}, H_{13}\right\}$ and $\mathcal{A}_{H_{4} \cap H_{56}}=\left\{H_{4}, H_{56}\right\}$ shows that $H_{4} \notin \pi_{1} \cup \pi_{2} \cup \pi_{3} \cup \pi_{4}=\mathcal{A}$ which is absurd. We conclude that $\mathcal{A}$ is not nice.

\subsection{Proof of Theorem 1.5.}

Thanks to Corollary 2.32, the question of the presence of a hereditary (inductive) factorization reduces to the case when $\mathcal{A}$ is irreducible. Thus we may assume that $W$ is irreducible.

The reverse implication of Theorem 1.5 is clear. So suppose that $W$ is irreducible so that $\mathcal{A}=\mathcal{A}(W)$ is nice. We need to show that $\mathcal{A}^{X}$ is also nice for every $X \in L(\mathcal{A})$. If $\mathcal{A}$ is supersolvable, then so is $\mathcal{A}^{X}$ for every $X \in L(\mathcal{A})$, by [Sta72, Prop. 3.2]. Consequently, $\mathcal{A}^{X}$ is factored again, thanks to Proposition 2.18. For $W$ of rank 3 , the result follows from Lemma 2.31. Thus, Theorem 1.5 follows from Theorem 1.3.

Finally, we comment on questions of computations underlying this work.

Remark 3.11. In order to establish several of our results we first use the functionality for complex reflection groups provided by the CHEVIE package in GAP (and some GAP code by J. Michel [M15]) (see $\left[\mathrm{S}^{+} 97\right]$ and $\left[\mathrm{GHL}^{+} 96\right]$ ) in order to obtain explicit linear functionals $\alpha$ defining the hyperplanes $H=\operatorname{ker} \alpha$ of the reflection arrangement $\mathcal{A}(W)$. We then use the functionality of SAGE $\left(\left[\mathrm{S}^{+} 09\right]\right)$ to explicitly determine the intersection lattice $L(\mathcal{A}(W))$ in the relevant instances, see Lemmas 3.5 - 3.10. 


\section{References}

[AHR14] N. Amend, T. Hoge and G. Röhrle, On inductively free restrictions of reflection arrangements, J. Algebra 418 (2014), 197-212.

[BC12] M. Barakat and M. Cuntz, Coxeter and crystallographic arrangements are inductively free, Adv. Math 229 (2012), 691-709.

[Bou68] N. Bourbaki, Éléments de mathématique. Groupes et algèbres de Lie. Chapitre IV-VI, Actualités Scientifiques et Industrielles, No. 1337, Hermann, Paris, 1968.

$\left[\mathrm{GHL}^{+} 96\right]$ M. Geck, G. Hiß, F. Lübeck, G. Malle, and G. Pfeiffer, CHEVIE - A system for computing and processing generic character tables, Appl. Algebra Engrg. Comm. Comput. 7 (1996), 175-210.

[HR14] T. Hoge and G. Röhrle, On supersolvable reflection arrangements, Proc. AMS, 142 (2014), no. 11, 3787-3799.

[HR15]_ On inductively free reflection arrangements, J. Reine u. Angew. Math. 701 (2015), 205-220.

[HR16] _ Addition-Deletion Theorems for Factorizations of Orlik-Solomon Algebras and nice Arrangements, European J. Combin. 55 (2016), 20-40.

[J90] M. Jambu, Fiber-type arrangements and factorization properties. Adv. Math. 80 (1990), no. 1, 1-21.

[JP95] M. Jambu and L. Paris, Combinatorics of Inductively Factored Arrangements, European J. Combin. 16 (1995), 267-292.

[JT84] M. Jambu and H. Terao, Free arrangements of hyperplanes and supersolvable lattices, Adv. in Math. 52 (1984), no. 3, 248-258.

[M15] J. Michel. The development version of the CHEVIE package of GAP3. J. Algebra, 435 (2015), 308-336.

[OS80] P. Orlik and L. Solomon, Combinatorics and topology of complements of hyperplanes, Invent. math. 56 (1980), 77-94.

[OS82] _ Arrangements Defined by Unitary Reflection Groups, Math. Ann. 261, (1982), 339-357.

[OST84] P. Orlik, L. Solomon, and H. Terao, Arrangements of hyperplanes and differential forms. Combinatorics and algebra (Boulder, Colo., 1983), 29-65, Contemp. Math., 34, Amer. Math. Soc., Providence, RI, 1984.

[OT92] P. Orlik and H. Terao, Arrangements of hyperplanes, Springer-Verlag, 1992.

[S+97] M. Schönert et al., GAP - Groups, Algorithms, and Programming - version 3 release 4, 1997.

[ST54] G.C. Shephard and J.A. Todd, Finite unitary reflection groups. Canadian J. Math. 6, (1954), 274-304. 
[S+09] W. A. Stein et al., Sage Mathematics Software, The Sage Development Team, 2009, http: //www. sagemath.org.

[Sta72] R. P. Stanley, Supersolvable lattices, Algebra Universalis 2 (1972), 197-217.

[Ste60] R. Steinberg, Invariants of finite reflection groups, Canad. J. Math. 12, (1960), 616-618.

[Ter80] H. Terao, Arrangements of hyperplanes and their freeness I, J. Fac. Sci. Univ. Tokyo 27 (1980), 293-320.

[Ter81] _ Generalized exponents of a free arrangement of hyperplanes and Shepherd-Todd-Brieskorn formula, Invent. Math. 63 no. 1 (1981) 159-179.

[Ter92] _ Factorizations of the Orlik-Solomon Algebras, Adv. in Math. 92, (1992), 45-53. 